\title{
Supporting Information Total Synthesis of Ningalin D
}

\author{
Akiyuki Hamasaki, Jeffrey M. Zimpleman, Inkyu Hwang and Dale L. Boger
}

Department of Chemistry and the Skaggs Institute for Chemical Biology, The Scripps Research Institute, 10550 North Torrey Pines Road, La Jolla, California 92037

$\overbrace{\mathrm{Br}}^{\mathrm{MeO}} \overbrace{\mathrm{CO}_{2} \mathrm{Me}}$

Methyl 2-Bromo-4,5-dimethoxyphenylacetate. A solution of 2-bromo-4,5-dimethoxyphenylacetonitrile ${ }^{8}$ (25 g, $97.7 \mathrm{mmol}$ ) and $\mathrm{H}_{2} \mathrm{SO}_{4}(10.4 \mathrm{~mL}, 195$ mmol) in $\mathrm{MeOH}(21 \mathrm{~mL})$ was warmed at reflux $\left(65^{\circ} \mathrm{C}\right)$ overnight (no nitrile absorption in IR). The cooled mixture was diluted with $\mathrm{H}_{2} \mathrm{O}(54 \mathrm{~mL})$ and treated with aqueous $10 \%$ $\mathrm{NaHCO}_{3}$ until the solution was basic. The mixture was extracted with $\mathrm{CH}_{2} \mathrm{Cl}_{2}$ and the combined organic extracts were washed with saturated aqueous $\mathrm{NaCl}$ and dried $\left(\mathrm{MgSO}_{4}\right)$. Removal of the solvent in vacuo afford the title compound (25.4 g, 90\%) identical in all respects $\left({ }^{1} \mathrm{H}\right.$ NMR $\left.,{ }^{13} \mathrm{C} \mathrm{NMR}, \mathrm{mp}\right)$ with that previously reported. ${ }^{9}$

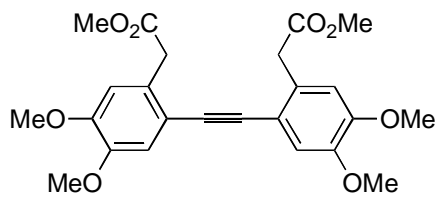

(3)

A solution of methyl 2-bromo-4,5-dimethoxyphenylacetate ${ }^{9}$ (5.26 g, 18.2 mmol, 2.2 equiv), bis(tributylstannyl)acetylene $(5.00 \mathrm{~g}, 8.28 \mathrm{mmol})$ and $\mathrm{Pd}\left(\mathrm{PPh}_{3}\right)_{4}(957 \mathrm{mg}, 0.828 \mathrm{mmol}, 10$ $\mathrm{mol} \%$ ) in $50 \mathrm{~mL}$ of degassed toluene was warmed at reflux for $6 \mathrm{~h}$ before the cooled reaction mixture was filtered through Celite. Removal of the solvent and chromatography $\left(\mathrm{SiO}_{2}, 4\right.$ $\times 15 \mathrm{~cm}, 50 \%$ EtOAc/hexane) afforded 4 (2.60 g, $5.88 \mathrm{mmol}, 71 \%$; typically $70-80 \%)$ as a colorless solid: mp 146-148 ${ }^{\circ} \mathrm{C} ;{ }^{1} \mathrm{H}$ NMR $\left(\mathrm{CDCl}_{3}, 400 \mathrm{MHz}\right) \delta 7.02(\mathrm{~s}, 2 \mathrm{H}), 6.80(\mathrm{~s}, 2 \mathrm{H})$, 3.89 (s, 12H), 3.85 (s, 4H), $3.68(\mathrm{~s}, 6 \mathrm{H}) ;{ }^{13} \mathrm{C} \mathrm{NMR}\left(\mathrm{CDCl}_{3}, 100 \mathrm{MHz}\right) \delta 171.8,149.3,147.8$, 
129.0, 115.5, 114.3, 112.6, 90.4, 55.93, 55.88, 52.0, 39.3; IR (film) $v_{\max } 2953,1737,1522$, $1358,1329,1238,1008 \mathrm{~cm}^{-1}$; ESI-TOF HRMS $\mathrm{m} / z 443.1697\left(\mathrm{C}_{24} \mathrm{H}_{26} \mathrm{O}_{8}+\mathrm{H}^{+}\right.$requires 443.1700).

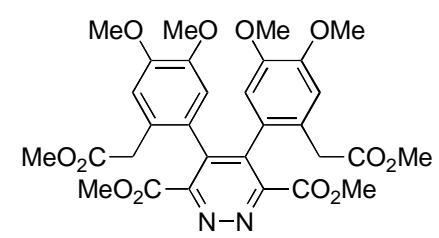

\section{(4)}

A solution of dimethyl 1,2,4,5-tetrazine-3,6-dicarboxylate ${ }^{10}(\mathbf{2}, 888 \mathrm{mg}, 4.48 \mathrm{mmol}, 1.2$ equiv) and acetylene $3(1.65 \mathrm{~g}, 3.73 \mathrm{mmol})$ in toluene $(15 \mathrm{~mL})$ was warmed at reflux $\left(110^{\circ} \mathrm{C}\right)$ for $13 \mathrm{~h}$ before the reaction mixture was cooled to $25^{\circ} \mathrm{C}$. Additional tetrazine $2(371 \mathrm{mg}$, $1.87 \mathrm{mmol}, 0.5$ equiv) was added and the mixture was warmed at reflux $\left(110{ }^{\circ} \mathrm{C}\right)$ for an additional $23 \mathrm{~h}$. Removal of the solvent and chromatography $\left(\mathrm{SiO}_{2}, 3 \times 20 \mathrm{~cm}, 67 \%\right.$ EtOAc/hexane) afforded 5 as a yellow solid (1.98 g, $3.23 \mathrm{mmol}, 87 \%)$ as a mixture of atropisomers (major isomer:minor isomer $=2.5: 1): \mathrm{mp} 161.2-162.0{ }^{\circ} \mathrm{C}(\mathrm{EtOAc}) ;{ }^{1} \mathrm{H}$ NMR $\left(\mathrm{CDCl}_{3}, 400 \mathrm{MHz}\right)$ major isomer: $\delta 6.73(\mathrm{~s}, 2 \mathrm{H}), 6.42(\mathrm{~s}, 2 \mathrm{H}), 3.83(\mathrm{~s}, 6 \mathrm{H}), 3.77(\mathrm{~s}, 6 \mathrm{H}), 3.65$ (s, $6 \mathrm{H}), 3.56(\mathrm{~s}, 6 \mathrm{H}), 3.27(\mathrm{~d}, 2 \mathrm{H}, J=16.0 \mathrm{~Hz}), 3.21(\mathrm{~d}, 2 \mathrm{H}, J=16.0 \mathrm{~Hz})$; minor isomer: $\delta$ 6.76 (s, 2H), 6.44 (s, 2H), 3.84 (s, 6H), 3.76 (s, 6H), 3.64 (s, 6H), 3.61 (s, 6H), 3.27 (d, 2H, J $=16.0 \mathrm{~Hz}), 3.21(\mathrm{~d}, 2 \mathrm{H}, J=16.0 \mathrm{~Hz}) ;{ }^{13} \mathrm{C} \mathrm{NMR}\left(\mathrm{CDCl}_{3}, 100 \mathrm{MHz}\right)$ major isomer: $\delta 170.8$, 164.7, 154.6, 149.4, 147.7, 138.7, 124.4, 123.9, 112.8, 112.7, 56.0, 55.7, 53.2, 52.0, 38.0; minor isomer: $\delta 170.8,164.7,154.8,149.3,147.4,138.6,124.2,123.5,112.9,111.8,55.8$, 55.7, 53.4, 52.0, 37.3; IR (film) $v_{\max } 1733 \mathrm{~cm}^{-1}$; MALDIFTMS (DHB) $\mathrm{m} / z 613.2028$ $\left(\mathrm{C}_{30} \mathrm{H}_{32} \mathrm{~N}_{2} \mathrm{O}_{12}+\mathrm{H}^{+}\right.$requires 613.2026).

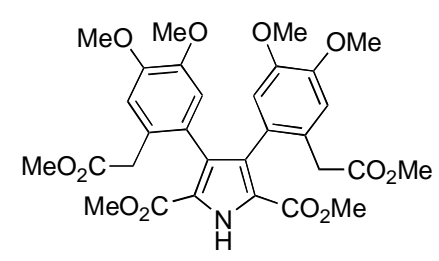

(5) 
A solution of $4(2.19 \mathrm{~g}, 3.58 \mathrm{mmol})$ in $\mathrm{CH}_{2} \mathrm{Cl}_{2}(15 \mathrm{~mL})$ was treated with activated $\mathrm{Zn}(3.51 \mathrm{~g}$, $53.7 \mathrm{mmol}, 15$ equiv) and trifluoroacetic acid $(8.2 \mathrm{~mL}, 107 \mathrm{mmol}, 30$ equiv) and the mixture was stirred at $25^{\circ} \mathrm{C}$. After $1 \mathrm{~h}$, additional $\mathrm{Zn}(3.51 \mathrm{~g}, 53.7 \mathrm{mmol}, 15$ equiv) was added and the mixture was stirred at $25^{\circ} \mathrm{C}$ for an additional $6 \mathrm{~h}$. The reaction mixture was neutralized with the addition of saturated aqueous $\mathrm{NaHCO}_{3}$, filtered through Celite, and extracted with $\mathrm{CH}_{2} \mathrm{Cl}_{2}(3 \times)$. The combined organic layer was dried over $\mathrm{Na}_{2} \mathrm{SO}_{4}$ and evaporated in vacuo. Chromatography $\left(\mathrm{SiO}_{2}, 2 \times 20 \mathrm{~cm}, 33 \% \mathrm{EtOAc} / \mathrm{CH}_{2} \mathrm{Cl}_{2}\right)$ afforded $6(1.37 \mathrm{~g}, 2.28 \mathrm{mmol}$, $64 \%$ ) as a yellow amorphous solid. The product was obtained as 2:1 mixture of atropisomers: mp 166.3-167.6 ${ }^{\circ} \mathrm{C}$ (EtOAc); ${ }^{1} \mathrm{H}$ NMR $\left(\mathrm{CDCl}_{3}, 400 \mathrm{MHz}\right)$ major isomer: $\delta 9.90$ (brs, $\left.1 \mathrm{H}\right)$, $6.79(\mathrm{~s}, 2 \mathrm{H}), 6.37$ (s, 2H), 3.84 (s, 6H), 3.74 (s, 6H), 3.58 (s, 6H), 3.57 (s, 6H), 3.35 (d, 2H, $J$ $=15.5 \mathrm{~Hz}), 3.24(\mathrm{~d}, 2 \mathrm{H}, J=15.9 \mathrm{~Hz})$; minor isomer: $\delta 9.93(\mathrm{brs}, 1 \mathrm{H}), 6.78(\mathrm{~s}, 2 \mathrm{H}), 6.56(\mathrm{~s}$, 2H), $3.84(\mathrm{~s}, 6 \mathrm{H}), 3.72(\mathrm{~s}, 6 \mathrm{H}), 3.66(\mathrm{~s}, 6 \mathrm{H}), 3.57(\mathrm{~s}, 6 \mathrm{H}), 3.35(\mathrm{~d}, 2 \mathrm{H}, J=15.5 \mathrm{~Hz}), 3.24(\mathrm{~d}$, $2 \mathrm{H}, J=15.9 \mathrm{~Hz}) ;{ }^{13} \mathrm{C} \mathrm{NMR}\left(\mathrm{CDCl}_{3}, 150 \mathrm{MHz}\right)$ major isomer: $\delta 172.1,160.42,148.16,147.3$, $129.9,125.8,125.0,122.3,113.8,112.0,55.80,55.73,51.9,51.72,38.3$; minor isomer: $\delta$ 172.0, 160.44, 148.21, 146.9, 129.7, 125.6, 124.7, 122.5, 114.5, 112.4, 55.8, 55.65, 51.9, 51.7, 37.8; IR (film) $v_{\max } 3445,1733,1715 \mathrm{~cm}^{-1}$; MALDIFTMS (DHB) $\mathrm{m} / \mathrm{z} 622.1908$ $\left(\mathrm{C}_{30} \mathrm{H}_{33} \mathrm{NO}_{12}+\mathrm{Na}^{+}\right.$requires 622.1895).

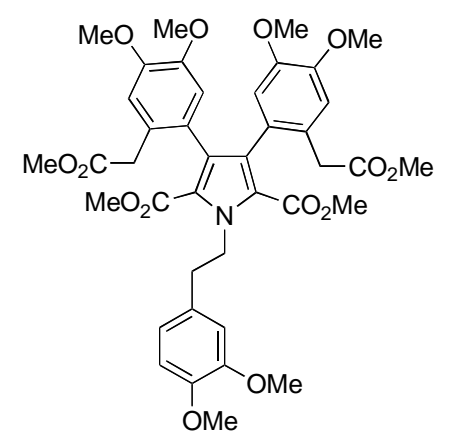

(7)

A stirred solution of $5(1.42 \mathrm{~g}, 2.37 \mathrm{mmol})$ in DMF $(7 \mathrm{~mL})$ was treated with 4-(2-iodoethyl)-1,2-dimethoxybenzene ${ }^{8 \mathrm{~b}}\left(\mathbf{6}, 2.08 \mathrm{~g}, 7.11 \mathrm{mmol}, 3\right.$ equiv) and $\mathrm{Cs}_{2} \mathrm{CO}_{3}(2.32 \mathrm{~g}$, $7.11 \mathrm{mmol}, 3$ equiv) at $25^{\circ} \mathrm{C}$. The mixture was stirred at $60^{\circ} \mathrm{C}$ for $2 \mathrm{~h}$ before being cooled to $25{ }^{\circ} \mathrm{C}$ and diluted with EtOAc. The mixture was washed with $\mathrm{H}_{2} \mathrm{O}$ and saturated aqueous 
$\mathrm{NaCl}$, and aqueous layer was back extracted with EtOAc. The combined organic layer was dried over $\mathrm{Na}_{2} \mathrm{SO}_{4}$, and the solvent was removed. Chromatography $\left(\mathrm{SiO}_{2}, 3 \times 20 \mathrm{~cm}, 67 \%\right.$ EtOAc/hexane) afforded $7(1.67 \mathrm{~g}, 2.19 \mathrm{mmol}, 92 \%)$ as a yellow amorphous solid. The product was obtained as 1.4:1 mixture of atropisomers: ${ }^{1} \mathrm{H} \mathrm{NMR}\left(\mathrm{CDCl}_{3}, 400 \mathrm{MHz}\right)$ major isomer: $\delta 6.85(\mathrm{~d}, 1 \mathrm{H}, J=1.4 \mathrm{~Hz}), 6.80(\mathrm{~s}, 2 \mathrm{H}), 6.72(\mathrm{~s}, 2 \mathrm{H}), 6.35(\mathrm{~s}, 2 \mathrm{H}), 5.12-4.96(\mathrm{~m}, 2 \mathrm{H})$, $3.91(\mathrm{~s}, 3 \mathrm{H}), 3.85$ (s, 3H), 3.83 (s, 6H), 3.65 (s, 6H), 3.61 (s, 6H), 3.49 (s, 6H), 3.42 (d, 2H, J $=15.3 \mathrm{~Hz}), 3.34(\mathrm{~d}, 2 \mathrm{H}, J=15.3 \mathrm{~Hz}), 3.10(\mathrm{t}, 2 \mathrm{H}, J=7.5 \mathrm{~Hz})$; minor isomer: $\delta 6.85(\mathrm{~d}, 1 \mathrm{H}, J$ $=1.4 \mathrm{~Hz}), 6.80(\mathrm{~s}, 2 \mathrm{H}), 6.71(\mathrm{~s}, 2 \mathrm{H}), 6.51(\mathrm{~s}, 2 \mathrm{H}), 5.12-4.96(\mathrm{~m}, 2 \mathrm{H}), 3.91(\mathrm{~s}, 3 \mathrm{H}), 3.85(\mathrm{~s}$, $3 \mathrm{H}), 3.83$ (s, 6H), 3.69 (s, 6H), $3.61(\mathrm{~s}, 6 \mathrm{H}), 3.49$ (s, 6H), $3.26(\mathrm{~d}, 2 \mathrm{H}, J=15.7 \mathrm{~Hz}), 3.19$ (d, $2 \mathrm{H}, J=15.8 \mathrm{~Hz}), 3.10(\mathrm{t}, 2 \mathrm{H}, J=7.5 \mathrm{~Hz}) ;{ }^{13} \mathrm{C} \mathrm{NMR}\left(\mathrm{CDCl}_{3}, 150 \mathrm{MHz}\right)$ major isomer: $\delta$ 172.07, 171.97, 161.4, 147.7, 146.9, 130.9, 129.6, 126.8, 125.3, 124.8, 121.2, 113.3, 112.5, 111.6, 111.2, 60.4, 55.90, 55.89, 55.78, 55.64, 51.77, 51.42, 48.56, 38.3, 37.80; minor isomer: $\delta 172.07,171.97,161.4,148.9,146.6,130.4,129.5,126.3,125.0,124.4,121.2,114.3$, $112.4,111.9,111.2,60.4,55.90,55.85,55.74,55.62,51.75,51.45,48.52,38.3,37.83$; IR (film) $v_{\max } 1738,1733 \mathrm{~cm}^{-1}$; MALDIFTMS (DHB) $\mathrm{m} / z .786 .2742\left(\mathrm{C}_{40} \mathrm{H}_{45} \mathrm{NO}_{14}+\mathrm{Na}^{+}\right.$requires 786.2732).

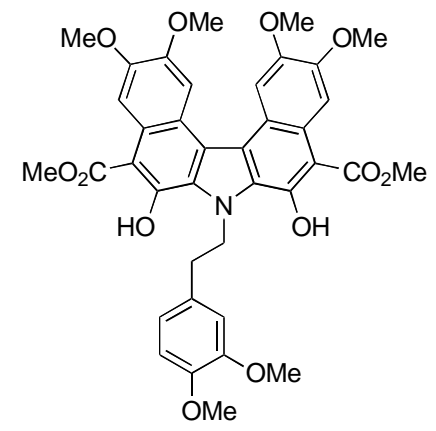

(8)

A solution of 7 (752 mg, $0.985 \mathrm{mmol})$ in DMF (7 mL) was treated with $\mathrm{NaH}(60 \%, 236 \mathrm{mg}$, $5.91 \mathrm{mmol}, 6$ equiv, washed with hexane and dried) and the mixture was stirred at $25^{\circ} \mathrm{C}$ for $15 \mathrm{~h}$, before being quenched by addition of aqueous $10 \% \mathrm{HCl}$. The precipitate was collected by filtration, dissolved in $\mathrm{CHCl}_{3}$ and dried over $\mathrm{Na}_{2} \mathrm{SO}_{4}$. Removal of the solvent gave 8 (559 $\mathrm{mg}, 0.799 \mathrm{mmol}, 81 \%)$ as a dark yellow solid: mp 241.0-243.5 ${ }^{\circ} \mathrm{C}$ (EtOAc); ${ }^{1} \mathrm{H}$ NMR 
$\left(\mathrm{CDCl}_{3}, 400 \mathrm{MHz}\right) \delta 13.27(\mathrm{~s}, 2 \mathrm{H}), 8.26(\mathrm{~s}, 2 \mathrm{H}), 8.10(\mathrm{~s}, 2 \mathrm{H}), 6.83(\mathrm{~m}, 2 \mathrm{H}), 6.76(\mathrm{~d}, 2 \mathrm{H}, J=$ $8.7 \mathrm{~Hz}), 5.47(\mathrm{t}, 2 \mathrm{H}, J=8.0 \mathrm{~Hz}), 4.18(\mathrm{~s}, 6 \mathrm{H}), 4.06(\mathrm{~s}, 6 \mathrm{H}), 3.86(\mathrm{~s}, 6 \mathrm{H}), 3.85(\mathrm{~s}, 3 \mathrm{H}), 3.82(\mathrm{~s}$, $3 \mathrm{H}), 3.21(\mathrm{t}, 2 \mathrm{H}, J=8.0 \mathrm{~Hz}) ;{ }^{13} \mathrm{C} \mathrm{NMR}\left(\mathrm{CDCl}_{3}, 100 \mathrm{MHz}\right) \delta 173.3,154.6,148.6,147.9,147.4$, 145.7, 131.4, 128.7, 122.3, 122.1, 120.9, 118.8, 112.1, 111.0, 107.3, 106.5, 102.5, 55.81, 55.77, 55.59, 55.55, 52.5, 49.5, 38.7; IR (film) $v_{\max } 2951,2834,1643,1515,1258 \mathrm{~cm}^{-1}$; MALDIFTMS (DHB) $m / z 699.2347\left(\mathrm{C}_{38} \mathrm{H}_{37} \mathrm{NO}_{12}\right.$ requires 699.2316).

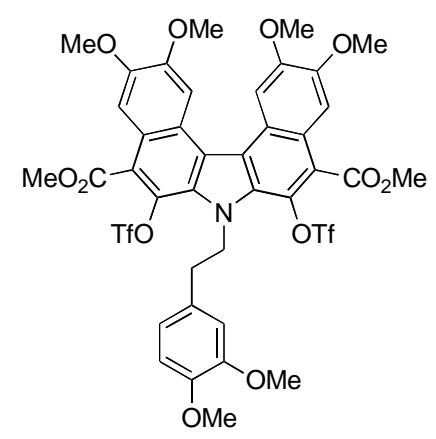

(9)

A solution of $\mathbf{8}(64 \mathrm{mg}, 0.091 \mathrm{mmol})$ in $\mathrm{CHCl}_{3}(0.45 \mathrm{~mL})$ and pyridine $(0.45 \mathrm{~mL})$ was treated with trifluoromethanesulfonic anhydride $\left(76.5 \mu \mathrm{L}, 0.455 \mathrm{mmol}, 5\right.$ equiv) at $0{ }^{\circ} \mathrm{C}$. The reaction mixture was allowed to warm to $25{ }^{\circ} \mathrm{C}$ with stirring for $2 \mathrm{~h}$. The reaction mixture was washed with aqueous $10 \% \mathrm{HCl}$, the organic layer was dried over $\mathrm{Na}_{2} \mathrm{SO}_{4}$ and the solvent was removed in vacuo. Chromatography $\left(\mathrm{SiO}_{2}, 1 \times 10 \mathrm{~cm}, 50 \%\right.$ EtOAc/hexane) afforded 9 (81 mg, $0.084 \mathrm{mmol}, 92 \%)$ as a tan solid: ${ }^{1} \mathrm{H} \mathrm{NMR}\left(\mathrm{CDCl}_{3}, 400 \mathrm{MHz}\right) \delta 7.91(\mathrm{~s}, 2 \mathrm{H}), 7.88(\mathrm{~s}$, $2 \mathrm{H}), 6.13(\mathrm{~d}, 1 \mathrm{H}, J=1.9 \mathrm{~Hz}), 5.91(\mathrm{~d}, 1 \mathrm{H}, J=8.2 \mathrm{~Hz}), 5.81(\mathrm{dd}, 1 \mathrm{H}, J=1.9,8.2 \mathrm{~Hz}), 5.08(\mathrm{t}$, $2 \mathrm{H}, J=6.3 \mathrm{~Hz}), 4.13(\mathrm{~s}, 6 \mathrm{H}), 4.08(\mathrm{~s}, 6 \mathrm{H}), 3.93(\mathrm{~s}, 6 \mathrm{H}), 3.52(\mathrm{~s}, 3 \mathrm{H}), 3.37(\mathrm{~s}, 3 \mathrm{H}), 2.73(\mathrm{t}, 2 \mathrm{H}$, $J=6.5 \mathrm{~Hz}) ;{ }^{13} \mathrm{C} \mathrm{NMR}\left(\mathrm{CDCl}_{3}, 150 \mathrm{MHz}, 50{ }^{\circ} \mathrm{C}\right) \delta 165.4,149.9,149.8,148.7,147.7,132.9$, 132.7, 129.1, 124.0, 123.3 (split), 121.8, 120.4, 119.7, 117.6, 111.4, 110.7, 106.5, 106.0, 56.3, 56.0, 55.5, 55.1, 52.9, 49.3, 35.7; IR (film) $v_{\max } 2955,1727,1598,1514,1427,1207,1159$, $1137 \mathrm{~cm}^{-1}$; ESI-TOF HRMS $m / z 964.1362\left(\mathrm{C}_{40} \mathrm{H}_{35} \mathrm{NO}_{16} \mathrm{~S}_{2}+\mathrm{H}^{+}\right.$requires 964.1374$)$. 


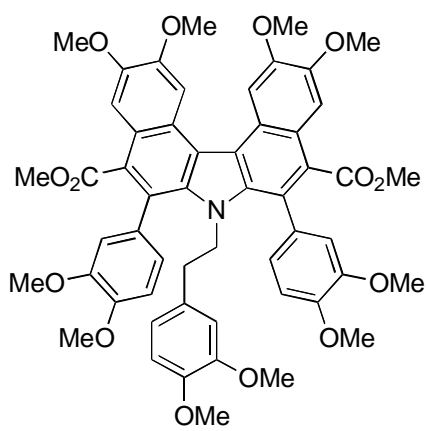

(11)

A solution of $\mathbf{9}(200 \mathrm{mg}, 0.208 \mathrm{mmol})$, 3,4-dimethoxyphenylboronic acid (10, $83 \mathrm{mg}, 0.458$ mmol, 2.2 equiv), $\mathrm{Pd}\left(\mathrm{PPh}_{3}\right)_{4}(24 \mathrm{mg}, 0.0208 \mathrm{mmol}, 10 \mathrm{~mol} \%)$ and $\mathrm{LiCl}(19 \mathrm{mg}, 0.458 \mathrm{mmol}$, 2.2 equiv) suspended in degassed DME $(1 \mathrm{~mL})$ was treated with $1 \mathrm{M}$ aqueous $\mathrm{K}_{2} \mathrm{CO}_{3}(0.83$ $\mathrm{mL}, 0.832 \mathrm{mmol}, 4$ equiv), and the mixture was stirred at $80{ }^{\circ} \mathrm{C}$ for $15 \mathrm{~h}$. The reaction mixture was diluted with EtOAc and saturated aqueous $\mathrm{NaHCO}_{3}$, and filtered through Celite. After phase separation and extraction of the aqueous phase with EtOAc $(2 \times)$, the combined organic layer was dried over $\mathrm{Na}_{2} \mathrm{SO}_{4}$ and evaporated. The residue and catechol (123 mg, $0.208 \mathrm{mmol}$ ) were dissolved in toluene-EtOAc, and the solution was evaporated to dryness. The residue was re-dissolved in toluene and evaporated. This re-dissolution and evaporation was repeated again, and the residue was purified by chromatography $\left(\mathrm{SiO}_{2}, 1 \times 25 \mathrm{~cm}\right.$, $67-75 \%$ EtOAc/hexane). A second chromatography $\left(\mathrm{SiO}_{2}, 1 \times 20 \mathrm{~cm}, 2.5 \% \mathrm{MeOH} / \mathrm{CH}_{2} \mathrm{Cl}_{2}\right)$ afforded pure 11 (172 mg, $0.183 \mathrm{mmol}, 88 \%)$ as dark yellow amorphous solid: ${ }^{1} \mathrm{H}$ NMR $\left(\mathrm{CDCl}_{3}, 600 \mathrm{MHz}, 50{ }^{\circ} \mathrm{C}\right) \delta 8.24(\mathrm{~s}, 2 \mathrm{H}), 7.32(\mathrm{~s}, 2 \mathrm{H}), 7.06(\mathrm{~d}, J=8.1 \mathrm{~Hz}, 2 \mathrm{H}), 7.04$ (brs, 2H), $6.88(\mathrm{~d}, J=8.0 \mathrm{~Hz}, 2 \mathrm{H}), 6.40(\mathrm{~d}, J=8.1 \mathrm{~Hz}, 1 \mathrm{H}), 5.88(\mathrm{~d}, J=8.1 \mathrm{~Hz}, 1 \mathrm{H}), 5.77(\mathrm{~s}, 1 \mathrm{H}), 4.04$ (s, 6H), $3.98(\mathrm{~s}, 6 \mathrm{H}), 3.89(\mathrm{~s}, 6 \mathrm{H}), 3.82(\mathrm{~s}, 6 \mathrm{H}), 3.69(\mathrm{~s}, 3 \mathrm{H}), 3.66(\mathrm{~s}, 6 \mathrm{H}), 3.55(\mathrm{~m}, 2 \mathrm{H}), 3.58$ (s, 3H), $2.11(\mathrm{~m}, 2 \mathrm{H}) ;{ }^{13} \mathrm{C} \mathrm{NMR}\left(150 \mathrm{MHz}, \mathrm{CDCl}_{3}, 50{ }^{\circ} \mathrm{C}\right) \delta 170.4,149.13,149.06,148.99$, $148.7,147.7,137.86,137.83,130.5,130.3,130.2$, 124.1, 123.5, 122.4, 121.7, 120.5, 120.4, 113.7, 112.1, 111.4, 111.3, 106.6, 105.5, 56.3, 56.1, 56.00, 55.97, 55.94, 55.7, 52.0, 46.7, 35.4; IR (film) $v_{\max } 1720,1510,1258 \mathrm{~cm}^{-1}$; UV (MeOH) $\lambda_{\max } 367$ (ع 22500), 331 (15900), 288 (49100); MALDIFTMS (DHB) $m / z, 962.3338\left(\mathrm{C}_{54} \mathrm{H}_{53} \mathrm{NO}_{14}+\mathrm{Na}^{+}\right.$requires 962.3358). 


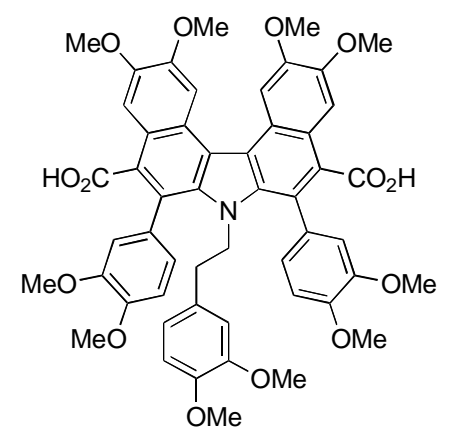

(12)

A solution of $11(65 \mathrm{mg}, 69.1 \mu \mathrm{mol}), t$-BuOK $(200 \mathrm{mg}, 1.78 \mathrm{mmol})$ and $\mathrm{H}_{2} \mathrm{O}(9.7 \mu \mathrm{L}, 0.538$ $\square \mathrm{mol})$ in DMSO $(0.5 \mathrm{~mL})$ was warmed at $80{ }^{\circ} \mathrm{C}$ and stirred for $24 \mathrm{~h}$. The reaction was quenched with the addition of $10 \%$ aqueous $\mathrm{HCl}$, and EtOAc was added to dissolve precipitate. After phase separation, the EtOAc layer was washed with $\mathrm{H}_{2} \mathrm{O}$ and saturated aqueous $\mathrm{NaCl}$, and dried over $\mathrm{Na}_{2} \mathrm{SO}_{4}$. Evaporation and chromatography $\left(\mathrm{SiO}_{2}, 1 \times 20 \mathrm{~cm}\right.$, $\left.20 \% \mathrm{MeOH} / \mathrm{CH}_{2} \mathrm{Cl}_{2}\right)$ afforded $12(53 \mathrm{mg}, 58.1 \mathrm{mmol}, 84 \%)$ as a tan solid: ${ }^{1} \mathrm{H} \mathrm{NMR}\left(\mathrm{CDCl}_{3}\right.$, $\left.600 \mathrm{MHz}, 50{ }^{\circ} \mathrm{C}\right) \delta 8.25(\mathrm{~s}, 2 \mathrm{H}), 7.47(\mathrm{~s}, 2 \mathrm{H}), 7.11(\mathrm{~d}, J=7.9 \mathrm{~Hz}, 2 \mathrm{H}), 7.08(\mathrm{brs}, 2 \mathrm{H}), 6.88(\mathrm{~d}$, $J=8.1 \mathrm{~Hz}, 2 \mathrm{H}), 6.38(\mathrm{~d}, J=8.2 \mathrm{~Hz}, 1 \mathrm{H}), 5.87(\mathrm{dd}, J=1.4,8.0 \mathrm{~Hz}, 1 \mathrm{H}), 5.77(\mathrm{~s}, 1 \mathrm{H}), 4.04$ (s, 6H), 3.99 (s, 6H), $3.87(\mathrm{~s}, 6 \mathrm{H}), 3.81(\mathrm{~s}, 6 \mathrm{H}), 3.66(\mathrm{~s}, 3 \mathrm{H}), 3.56(\mathrm{~m}, 2 \mathrm{H}), 3.54(\mathrm{~s}, 3 \mathrm{H}), 2.12(\mathrm{~m}$, $2 \mathrm{H}) ;{ }^{13} \mathrm{C}$ NMR $\left(150 \mathrm{MHz}, \mathrm{CD}_{3} \mathrm{OD}, 50{ }^{\circ} \mathrm{C}\right) \delta 173.6,150.9,150.6,150.3,149.99,149.95$, 149.0, 139.23, 139.21, 132.8, 132.0, 131.5, 124.7, 124.4, 122.8, 122.1, 121.1, 116.0, 113.6, 113.4, 113.1, 108.4, 107.4, 57.1, 56.9, 56.8, 56.7, 56.5, 56.4, 47.5, 36.5; IR (film) $v_{\max } 3514$, 3288, 2935, 2836, 1725, 1512, $1280 \mathrm{~cm}^{-1}$; UV (MeOH) $\lambda_{\max } 373$ (ع 19800), 328 (15600), 289 (47900); ESI-TOF HRMS $m / z 912.3223\left(\mathrm{C}_{52} \mathrm{H}_{49} \mathrm{NO}_{14}+\mathrm{H}^{+}\right.$requires 912.3226).

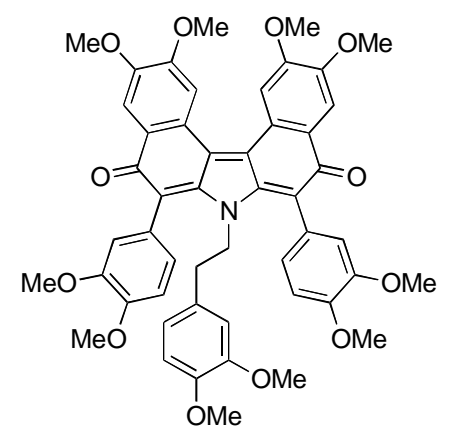




\section{permethyl ningalin D (13).}

A solution of $12(15 \mathrm{mg}, 16.4 \mu \mathrm{mol})$ in $\mathrm{CH}_{2} \mathrm{Cl}_{2}(0.3 \mathrm{~mL})$ was treated with DPPA $(17.7 \mu \mathrm{L}$, $82.0 \mu \mathrm{mol}, 5.0$ equiv) and $i$ - $\operatorname{Pr}_{2} \mathrm{NEt}\left(14.3 \mu \mathrm{L}, 82.0 \mu \mathrm{mol}, 5.0\right.$ equiv). After stirring at $25{ }^{\circ} \mathrm{C}$ for $20 \mathrm{~h}$, the mixture was washed with saturated aqueous $\mathrm{NaHCO}_{3}(2 \times)$ and saturated aqueous $\mathrm{NaCl}$. The organic layer was dried over $\mathrm{Na}_{2} \mathrm{SO}_{4}$, and solvent was removed. The residue was dissolved in THF $(0.3 \mathrm{~mL})$ and $\mathrm{H}_{2} \mathrm{O}(0.2 \mathrm{~mL})$, and warmed at reflux for $90 \mathrm{~h}$. The mixture was extracted with $\mathrm{CH}_{2} \mathrm{Cl}_{2}$ (4x, dried $\left(\mathrm{Na}_{2} \mathrm{SO}_{4}\right)$ and evaporated. Chromatography $\left(\mathrm{SiO}_{2}, 1 \times 15 \mathrm{~cm}, 2.5 \% \mathrm{MeOH} / \mathrm{CH}_{2} \mathrm{Cl}_{2}\right)$ gave pure $13(9.8 \mathrm{mg}, 11.5 \mu \mathrm{mol}, 70 \%)$ as a dark red amorphous solid: mp 258.0-260.2 ${ }^{\circ} \mathrm{C}\left(\mathrm{CH}_{2} \mathrm{Cl}_{2}\right) ;{ }^{1} \mathrm{H} \mathrm{NMR}\left(\mathrm{CDCl}_{3}, 400 \mathrm{MHz}\right) \delta 7.84(\mathrm{~s}, 2 \mathrm{H})$, 7.69 (s, 2H), 7.01-6.97 (m, 4H), 6.89 (d, $J=8.2 \mathrm{~Hz}, 2 \mathrm{H}), 6.39$ (d, $J=8.2 \mathrm{~Hz}, 1 \mathrm{H}), 6.16$ (dd, $J=1.9,8.1 \mathrm{~Hz}, 1 \mathrm{H}), 6.04(\mathrm{~d}, J=1.9 \mathrm{~Hz}, 1 \mathrm{H}), 4.02(\mathrm{~s}, 6 \mathrm{H}), 3.92(\mathrm{~s}, 6 \mathrm{H}), 3.90(\mathrm{~s}, 6 \mathrm{H}), 3.89(\mathrm{~s}$, $6 \mathrm{H}), 3.61(\mathrm{~s}, 3 \mathrm{H}), 3.60(\mathrm{~s}, 3 \mathrm{H}), 3.08(\mathrm{t}, J=7.5 \mathrm{~Hz}, 2 \mathrm{H}), 2.29(\mathrm{t}, J=7.3 \mathrm{~Hz}, 2 \mathrm{H}) ;{ }^{13} \mathrm{C} \mathrm{NMR}$ $\left(\mathrm{CDCl}_{3}, 100 \mathrm{MHz}\right) \delta 183.0,154.3,151.1,151.0,149.0,148.6,148.4,147.5,131.1,129.7$, $126.2,125.4,124.0,123.7,120.8,117.2$, 114.2, 111.7, 110.7, 110.6, 109.7 (split), 108.5 (split), 56.5 (split), 56.2 (split), 56.0 (split), 55.8 (split), 55.5 (split), 55.4 (split), 46.9, 34.0; IR (film) $v_{\max } 2917,1653,1507,1464,1261,1158,1026 \mathrm{~cm}^{-1}$; UV (MeOH) $\lambda_{\max } 501(\varepsilon$ 15000); ESI-TOF HRMS m/z, $854.3190\left(\mathrm{C}_{50} \mathrm{H}_{47} \mathrm{NO}_{12}+\mathrm{H}^{+}\right.$requires 854.3171).

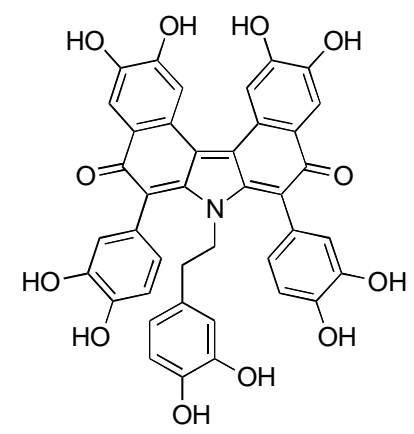

\section{ningalin D (1a).}

A solution of $13(14.1 \mathrm{mg}, 16.5 \mu \mathrm{mol})$ was treated with $1 \mathrm{M} \mathrm{BBr}_{3}$ in $\mathrm{CH}_{2} \mathrm{Cl}_{2}(0.248 \mathrm{~mL}, 248$ $\mu \mathrm{mol}, 15$ equiv) at $-78{ }^{\circ} \mathrm{C}$ and the mixture was allowed to warm to $25{ }^{\circ} \mathrm{C}$ over $16 \mathrm{~h}$. The reaction was quenched with the addition of $\mathrm{MeOH}(0.2 \mathrm{~mL})$, and solvents were removed under a $\mathrm{N}_{2}$ stream. The residue was dissolved in $\mathrm{H}_{2} \mathrm{O}(1.0 \mathrm{~mL})$ and extracted with EtOAc (1.0 
$\mathrm{mL}, 4 \times)$. The combined organic phase was dried $\left(\mathrm{Na}_{2} \mathrm{SO}_{4}\right)$ and evaporated. Chromatography (C18 reverse phase, $\left.1 \times 10 \mathrm{~cm}, 40 \% \mathrm{CH}_{3} \mathrm{CN} / 5 \% \mathrm{MeOH} / \mathrm{H}_{2} \mathrm{O}\right)$ afforded pure ningalin $\mathrm{D}(\mathbf{1 a})$ as a dark red amorphous solid $(11.3 \mathrm{mg}, 15.8 \mu \mathrm{mol}, 96 \%)$ identical in all respects with natural 1a: ${ }^{1} \mathrm{H}$ NMR $\left(\mathrm{CD}_{3} \mathrm{OD}, 400 \mathrm{MHz}\right) \delta 7.81(\mathrm{~s}, 2 \mathrm{H}), 7.41(\mathrm{~s}, 2 \mathrm{H}), 6.79-6.78$ (overlap of two doublets, $4 \mathrm{H}), 6.64(\mathrm{dd}, J=1.7,8.1 \mathrm{~Hz}, 2 \mathrm{H}), 6.26(\mathrm{~d}, J=8.0 \mathrm{~Hz}, 1 \mathrm{H}), 6.08(\mathrm{~d}, J=2.0 \mathrm{~Hz}, 1 \mathrm{H})$, $5.91(\mathrm{dd}, J=2.0,8.0 \mathrm{~Hz}, 1 \mathrm{H}), 2.91(\mathrm{t}, J=7.0 \mathrm{~Hz}, 2 \mathrm{H}), 2.09(\mathrm{t}, J=6.9 \mathrm{~Hz}, 2 \mathrm{H}) ;{ }^{13} \mathrm{C} \mathrm{NMR}$ $\left(\mathrm{CD}_{3} \mathrm{OD}, 150 \mathrm{MHz}\right) \delta 185.4,156.5,150.2,149.0,146.6,146.1,145.6,144.6,132.2,130.6$, 126.5, 126.1, 125.0, 124.2, 121.5, 119.4, 118.9, 117.2, 116.3, 115.7, 115.0, 114.2, 47.8, 35.0; IR (film) $v_{\max } 3700-3000,1759,1561,1296 \mathrm{~cm}^{-1}$; UV (MeOH) $\lambda_{\max } 507$ (ع 26800), 292 (32900); ESI-TOF HRMS $m / z 714.1596\left(\mathrm{C}_{40} \mathrm{H}_{27} \mathrm{NO}_{12}+\mathrm{H}^{+}\right.$requires 714.1606).

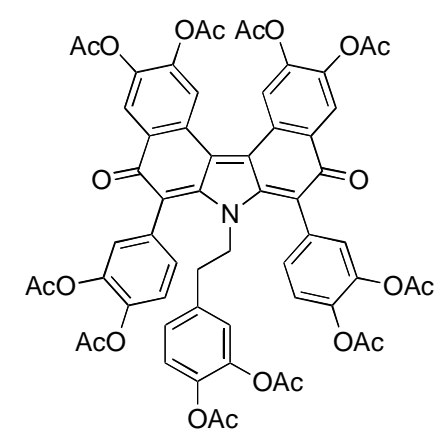

ningalin $D$ decaacetate (14).

A solution of ningalin $\mathrm{D}(\mathbf{1 a}, 7.4 \mathrm{mg}, 10.4 \mu \mathrm{mol})$ in pyridine $(0.2 \mathrm{~mL})$ was treated with acetic anhydride ( $98.3 \mu \mathrm{L}, 1.04 \mathrm{mmol}, 100$ equiv) at $25^{\circ} \mathrm{C}$. After $3 \mathrm{~h}$, the reaction mixture was diluted with EtOAc. The mixture was washed with aqueous $10 \% \mathrm{HCl}(2 \times)$ and saturated aqueous $\mathrm{NaHCO}_{3}(2 \times)$, dried $\left(\mathrm{Na}_{2} \mathrm{SO}_{4}\right)$ and evaporated. PTLC $\left(5 \% \mathrm{MeOH} / \mathrm{CH}_{2} \mathrm{Cl}_{2}\right)$ afforded ningalin D decaacetate (14) as a dark green amorphous solid (11.6 mg, $10.2 \mu \mathrm{mol}, 98 \%$ ) identical in all respects $\left({ }^{1} \mathrm{H}\right.$ NMR, HRMS) with the reported title compound: ${ }^{1} \mathrm{H}$ NMR $\left(\mathrm{CDCl}_{3}, 400 \mathrm{MHz}\right) \delta 8.17(\mathrm{~s}, 2 \mathrm{H}), 7.98(\mathrm{~s}, 2 \mathrm{H}), 7.29-7.23(\mathrm{~m}, 6 \mathrm{H}), 6.75(\mathrm{~d}, J=8.2 \mathrm{~Hz}, 1 \mathrm{H})$, $6.48(\mathrm{dd}, J=1.6,8.3 \mathrm{~Hz}, 1 \mathrm{H}), 6.43(\mathrm{~d}, J=1.5 \mathrm{~Hz}, 1 \mathrm{H}), 3.23(\mathrm{~m}, 2 \mathrm{H}), 2.35(\mathrm{~s}, 6 \mathrm{H}), 2.33$ (s, 6H), 2.30 (s, 6H), 2.29 (s, 6H), 2.24 (m, 2H), 2.14 (s, 3H), 2.13 (s, 3H); ${ }^{13} \mathrm{C} \mathrm{NMR}\left(\mathrm{CDCl}_{3}\right.$, $150 \mathrm{MHz}) \delta 181.0,168.1,167.88,167.86,167.80,167.77,167.72,154.2,144.6,144.1,142.1$, 
$141.7,141.5,140.7,136.1,131.2,130.3,129.9,129.0,128.9,127.6,127.0,126.5,123.6$, 123.4, 123.2, 122.0, 116.9, 53.4, 33.7, 20.8, 20.73, 20.68, 20.6, 20.5, 20.4; IR (film) $v_{\max }$ 2916, 1772, $1203 \mathrm{~cm}^{-1}$; UV (MeOH) $\lambda_{\max } 574$ ( $\varepsilon$ 5600), 468 (23300), 275 (sh); ESI-TOF HRMS $m / z, 1134.2648\left(\mathrm{C}_{60} \mathrm{H}_{47} \mathrm{NO}_{22}+\mathrm{H}^{+}\right.$requires 1134.2662).

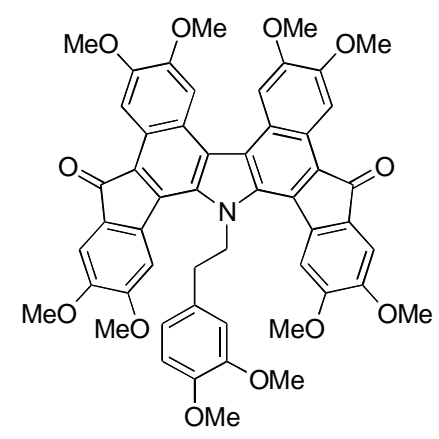

(15)

A solution of $12(15 \mathrm{mg}, 16.4 \mu \mathrm{mol})$ and $\mathrm{Et}_{3} \mathrm{~N}(6.9 \mu \mathrm{L}, 49.2 \mu \mathrm{mol}, 3$ equiv) was treated with $\mathrm{SOCl}_{2}$-DMF complex $\left(7.4 \mu \mathrm{L}, 49.2 \mu \mathrm{mol}, 3\right.$ equiv) at $25{ }^{\circ} \mathrm{C}$. After $2 \mathrm{~h}$, additional $\mathrm{SOCl}_{2}-\mathrm{DMF}$ complex ( $7.4 \mu \mathrm{L}, 49.2 \mu \mathrm{mol}, 3$ equiv) was added and the mixture was stirred at $25{ }^{\circ} \mathrm{C}$ for $2 \mathrm{~h}$. The mixture was basified by addition of saturated aqueous $\mathrm{NaHCO}_{3}$ and extracted with $\mathrm{CH}_{2} \mathrm{Cl}_{2}(3 \times)$. The combined $\mathrm{CH}_{2} \mathrm{Cl}_{2}$ layer was dried $\left(\mathrm{Na}_{2} \mathrm{SO}_{4}\right)$ and evaporated. PTLC (5\% $\mathrm{MeOH} / \mathrm{CH}_{2} \mathrm{Cl}_{2}$ ) afforded 15 as a black amorphous solid (10 mg, $11.4 \mu \mathrm{mol}$, 70\%): ${ }^{1} \mathrm{H}$ NMR $\left(\mathrm{CDCl}_{3}, 400 \mathrm{MHz}\right) \delta 8.57$ (s, 2H), $7.75(\mathrm{~s}, 2 \mathrm{H}), 7.32$ (s, 2H), $7.24(\mathrm{~s}, 2 \mathrm{H})$, $6.00-5.98(\mathrm{~m}, 1 \mathrm{H}), 5.92(\mathrm{dd}, J=1.9,8.1 \mathrm{~Hz}, 1 \mathrm{H}), 5.71(\mathrm{~d}, J=1.8 \mathrm{~Hz}, 1 \mathrm{H}), 4.91(\mathrm{t}, J=6.7 \mathrm{~Hz}$, 2H), 4.11 (s, 6H), 4.06 (s, 6H), $3.98(\mathrm{~s}, 6 \mathrm{H}), 3.93$ (s, 6H), 3.43 (s, 3H), 3.19 (s, 3H), 2.38 (t, $J$ $=6.6 \mathrm{~Hz}, 2 \mathrm{H}) ;{ }^{13} \mathrm{C} \mathrm{NMR}\left(\mathrm{CDCl}_{3}, 100 \mathrm{MHz}\right) \delta 194.3,153.0,150.8,149.1,148.8,147.8,147.0$, 140.4, 136.7, 131.0, 129.3, 128.4, 128.3, 126.4, 124.9, 124.1, 119.9, 111.2, 110.0 (split), 107.7 (split), 107.2 (split), 105.9 (split), 103.4, 56.3 (split), 56.1 (split), 55.9 (split), 55.6 (split), 55.2 (split), 54.7 (split), 51.8, 33.8; IR (film) $v_{\max }$ 2916, 1687, 1499, 1299, 1246, 1213, 1051, $1029 \mathrm{~cm}^{-1}$; UV $\left(\mathrm{CHCl}_{3}\right) \lambda_{\max } 572$ ( $\varepsilon$ 7600), 452 (10100), 370 (sh), 301 (59700); ESI-TOF HRMS $m / z 876.3005\left(\mathrm{C}_{52} \mathrm{H}_{45} \mathrm{NO}_{12}+\mathrm{H}^{+}\right.$requires 876.3014). 


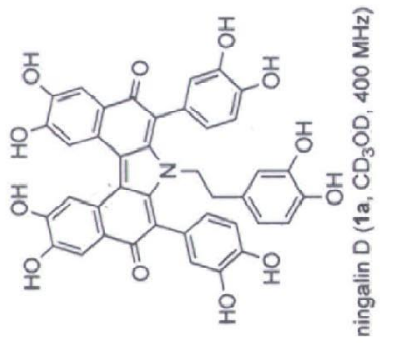

$\varepsilon 80$ 乙

$960 \mathrm{C}$

9012

$806 \mathrm{Z}$
$916 \mathrm{Z}$
$926 \mathrm{Z}$

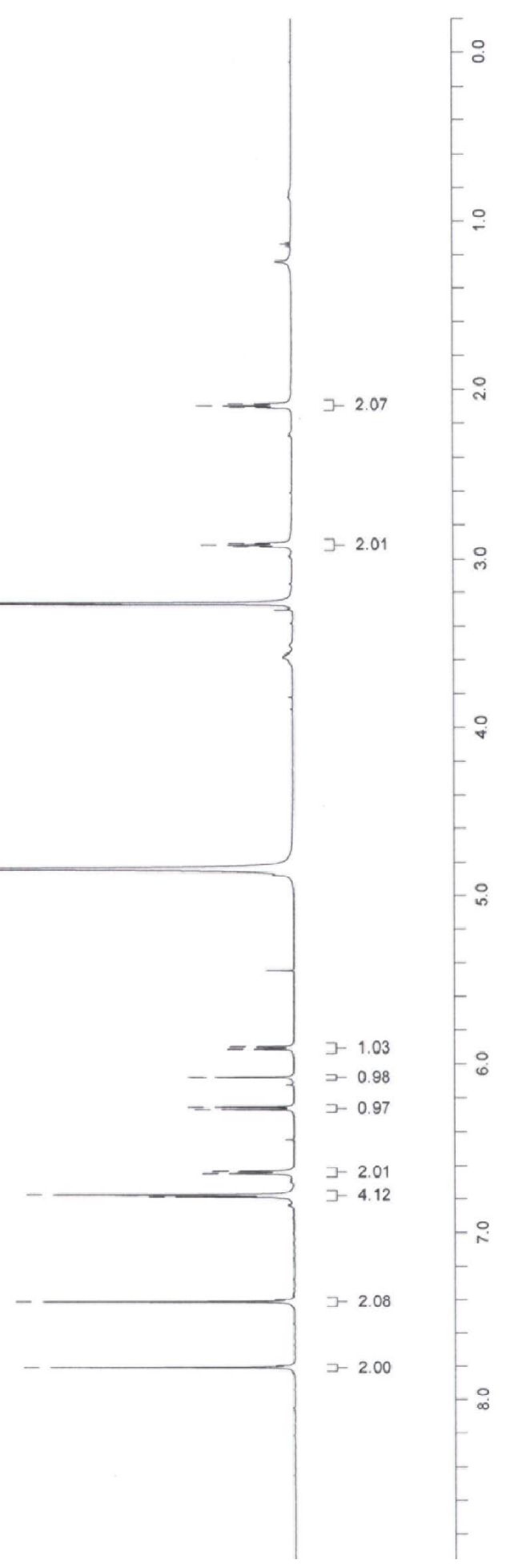



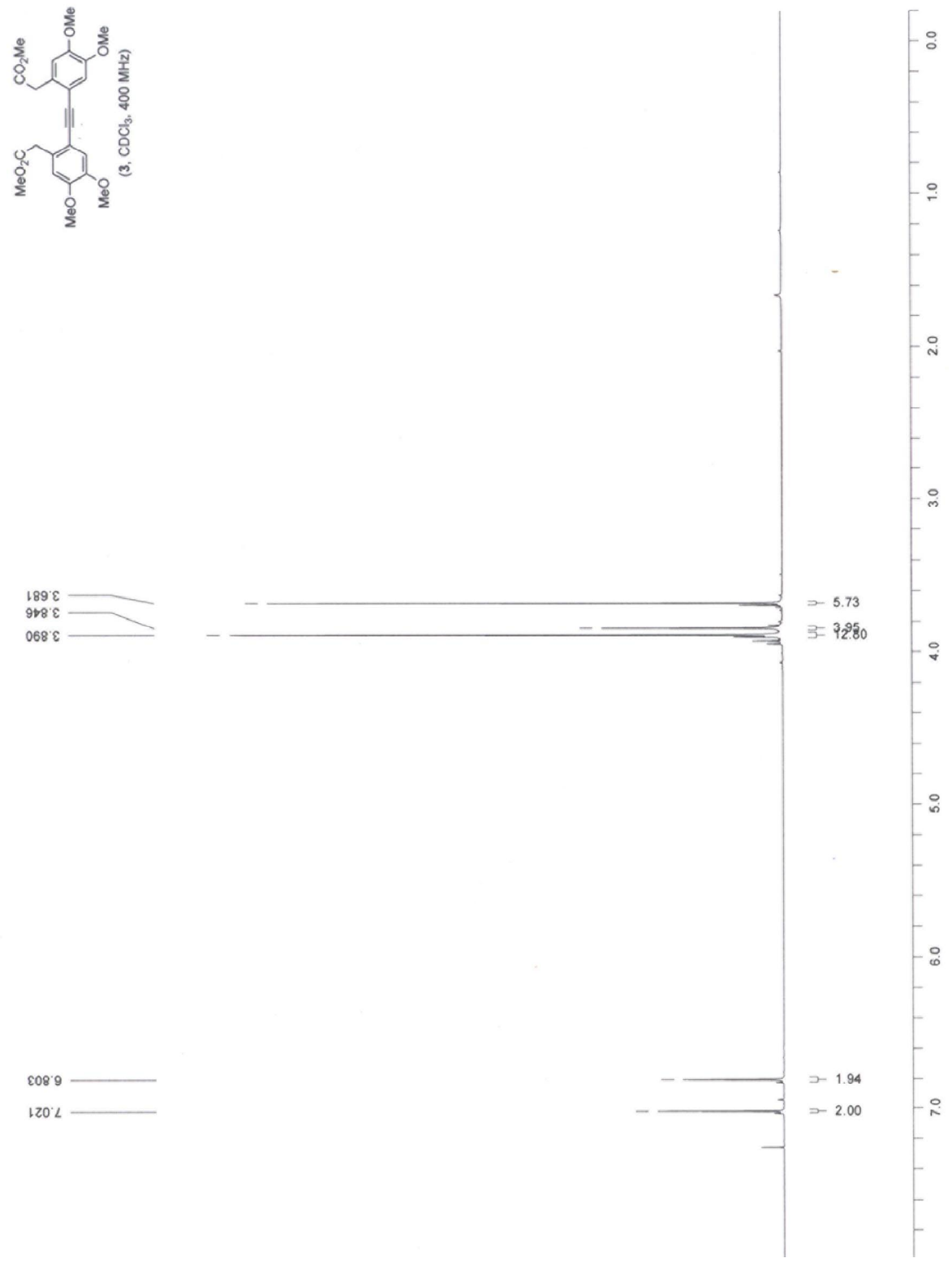


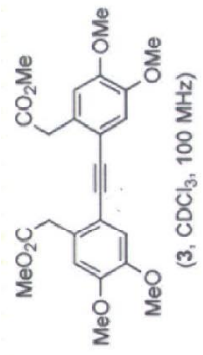

$80 \varepsilon^{\prime} 6 \varepsilon$



$89 L^{\circ} \angle t \mid$
$29 Z^{\prime} 6 b l$

LLS'ZLL
†०E'เ।L

เจ॰

696.821

เ88. 99

เE6 6

LSE 06

$008 \cdot \angle L$ 

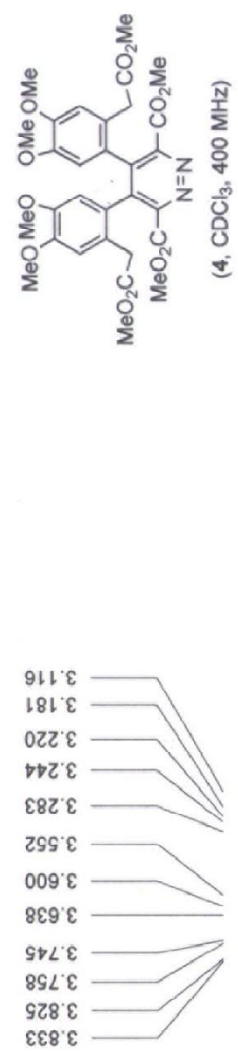

$8+5 \cdot 9$

LEt?

$62 L 9$
$67 L 9$

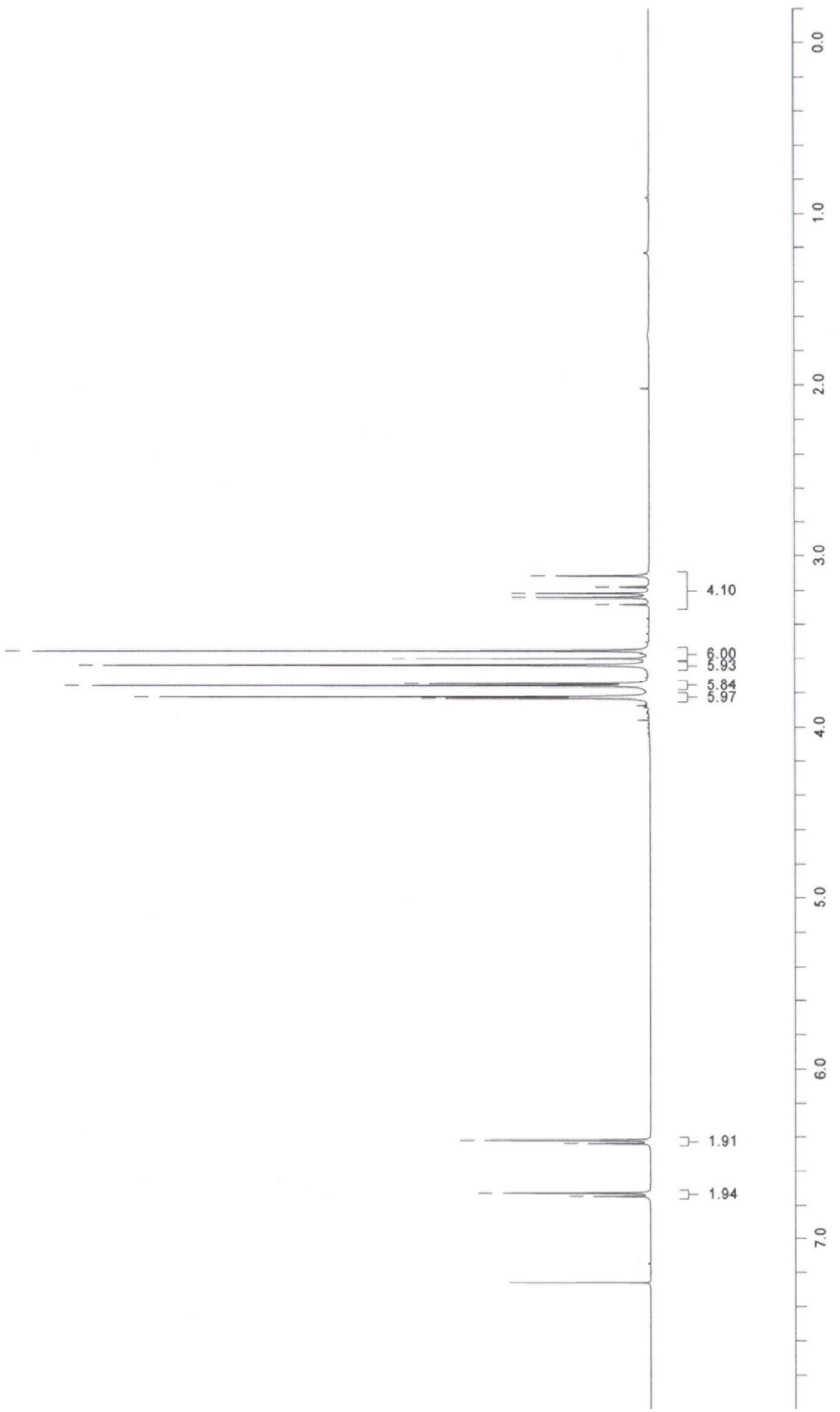



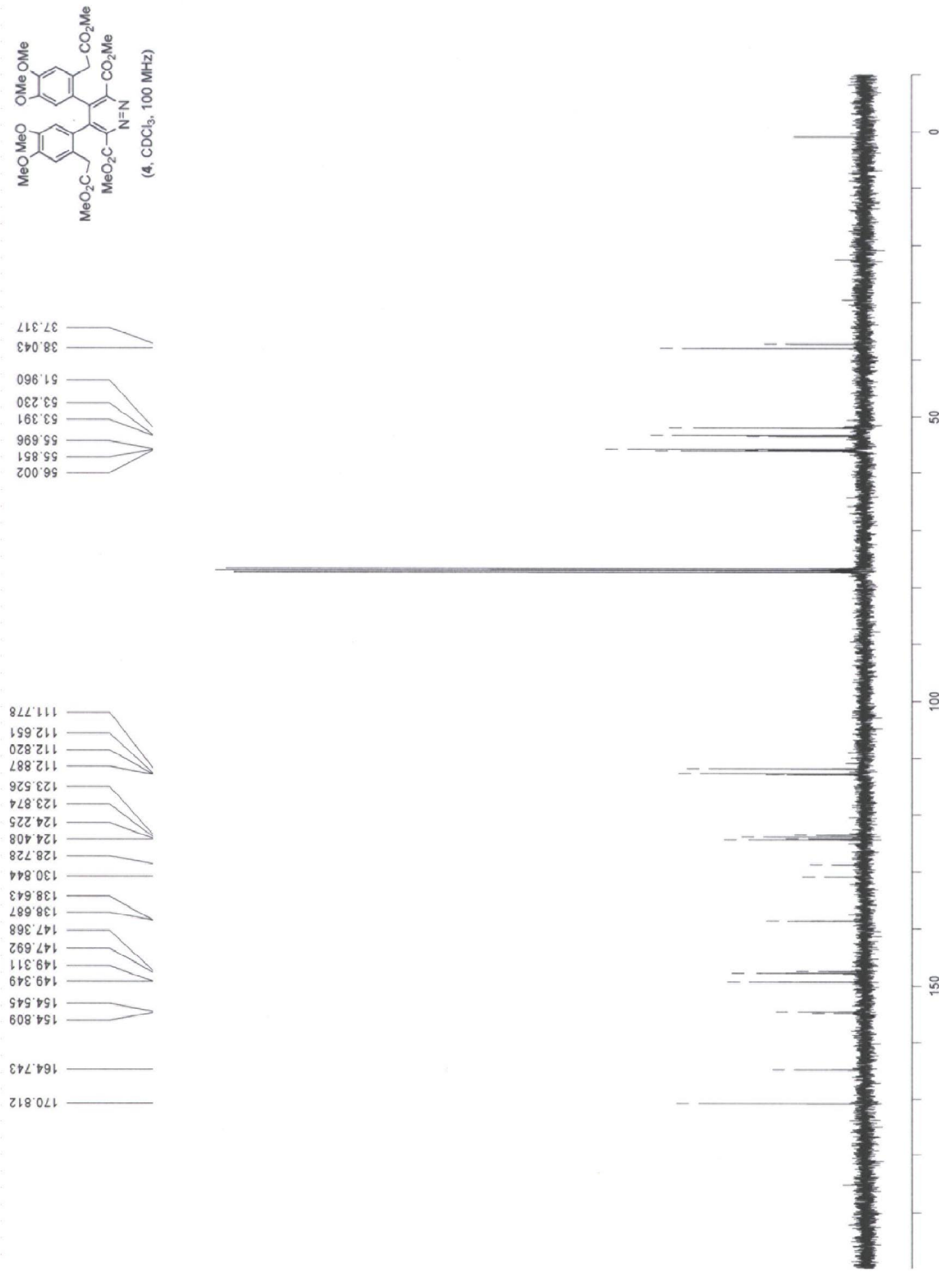

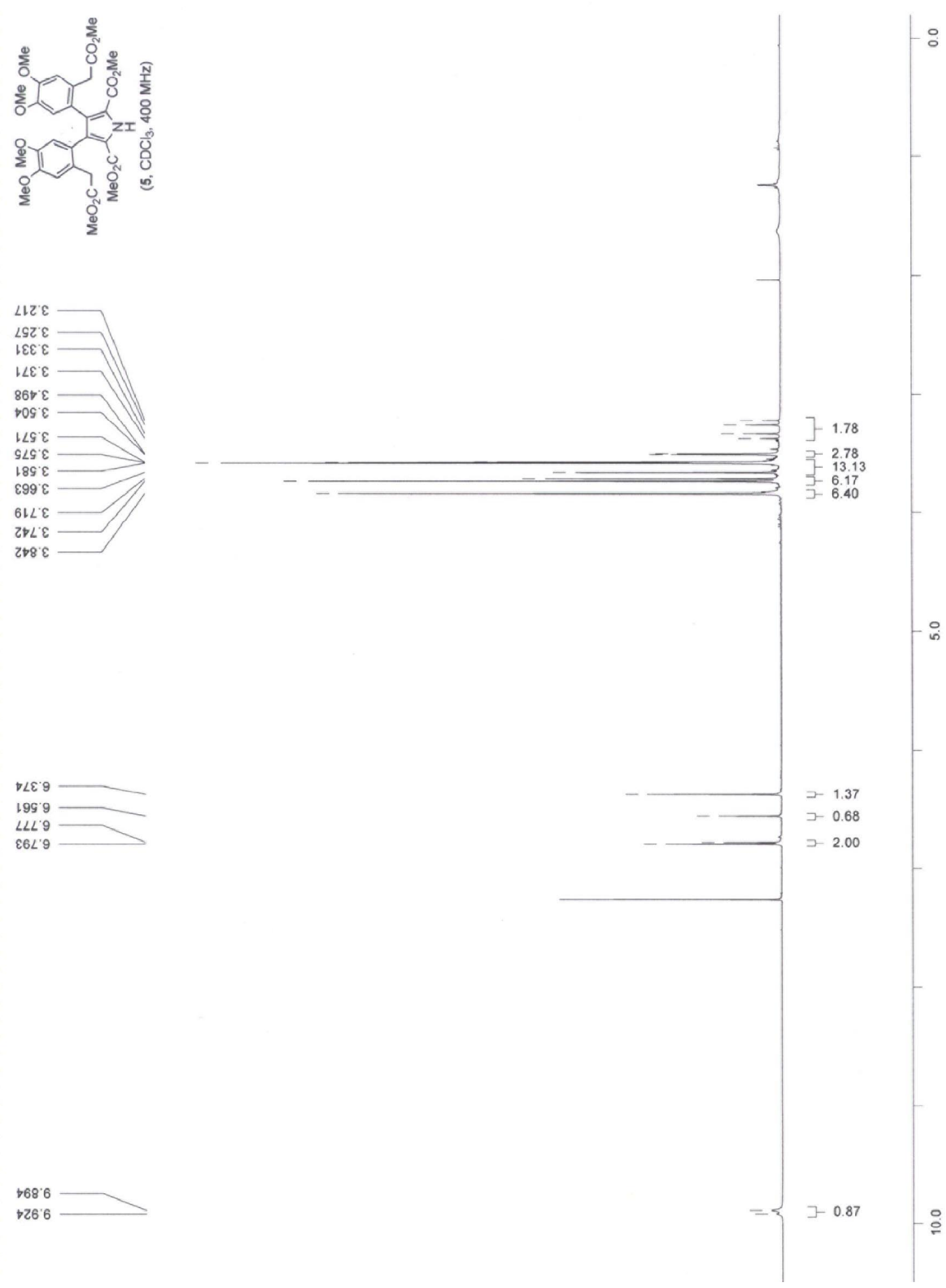

$\vdash 68^{\circ} 6$

$\rightarrow 26^{\prime} 6$

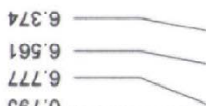

E6L'

S16 

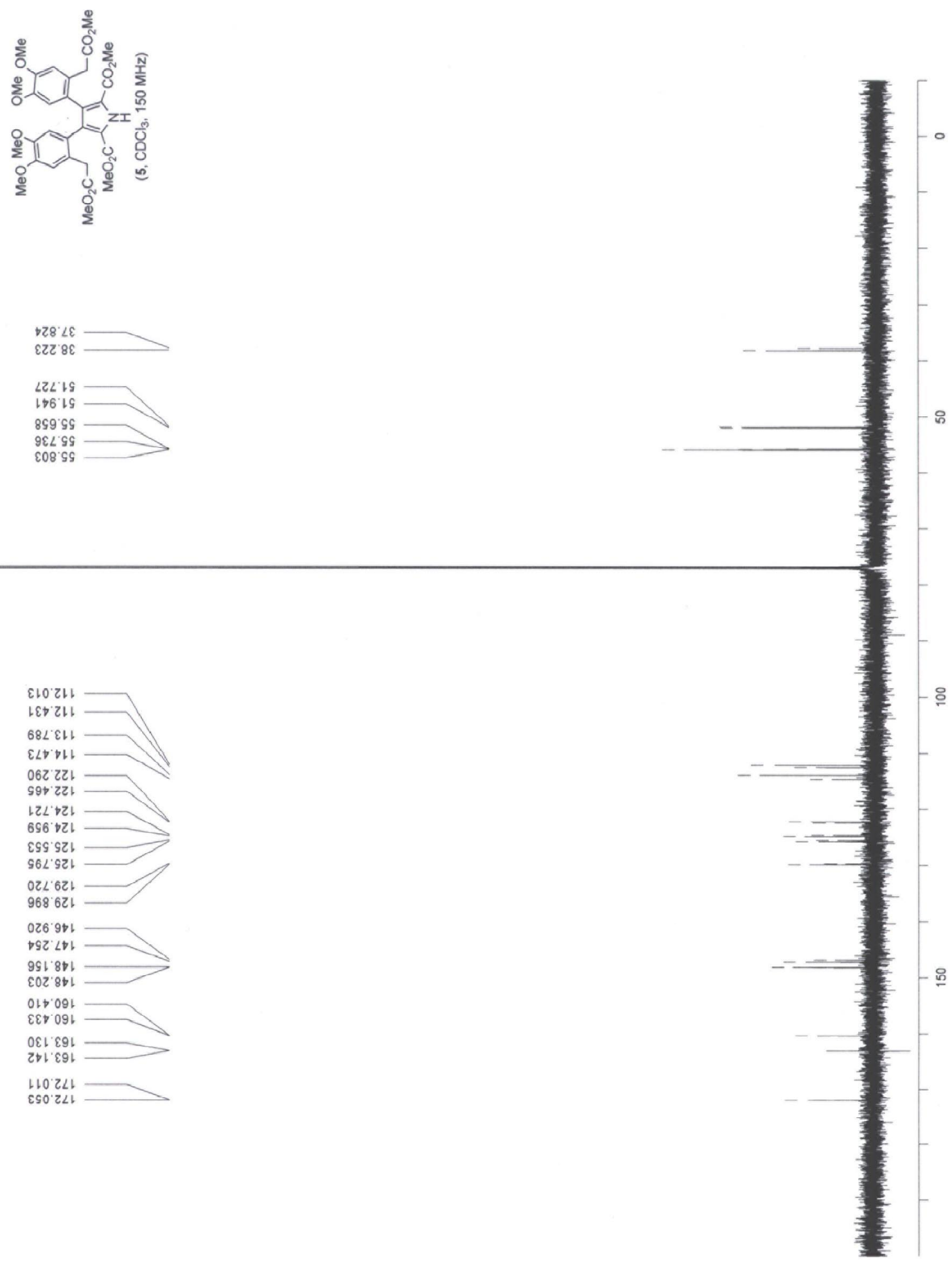

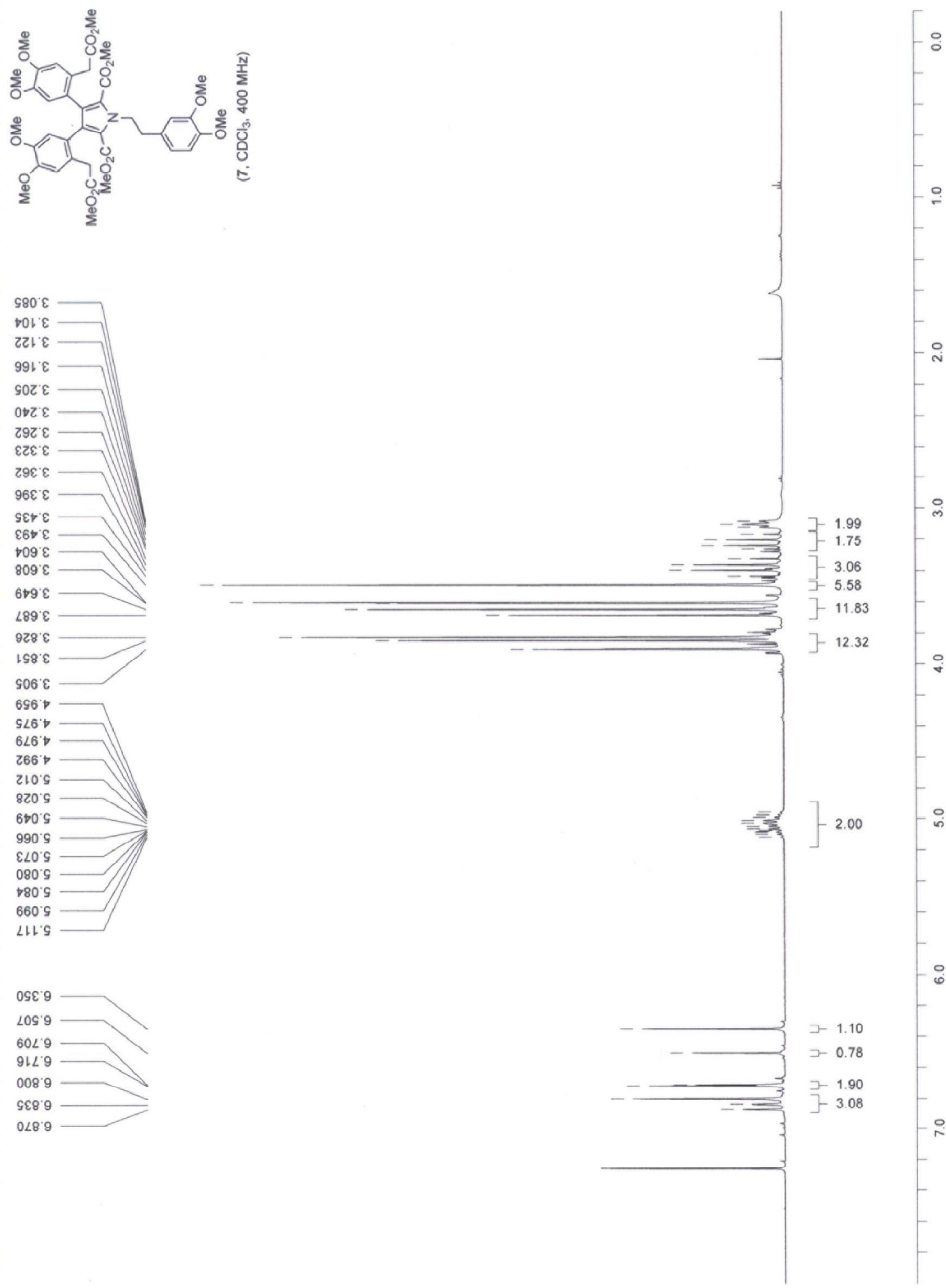

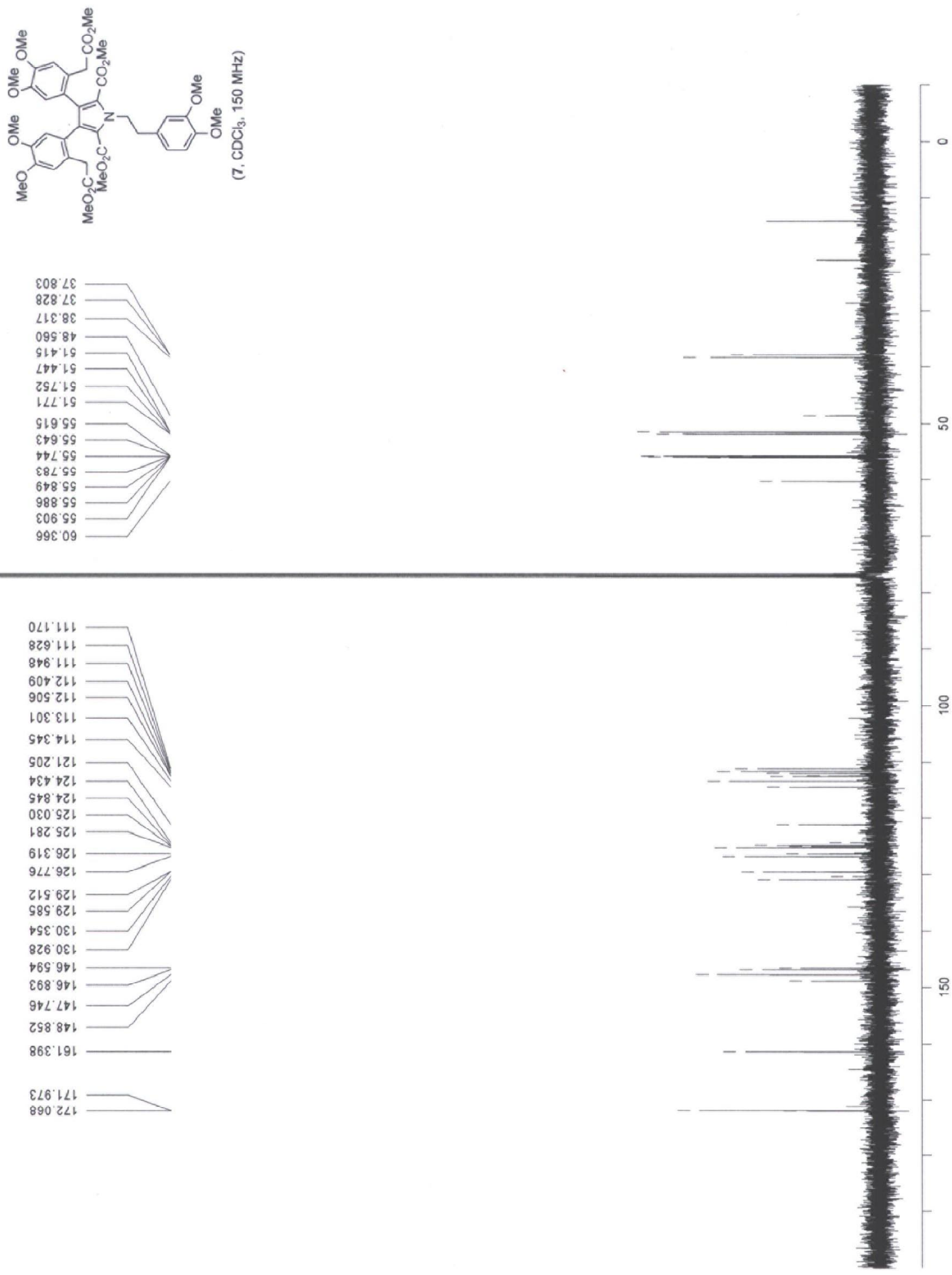

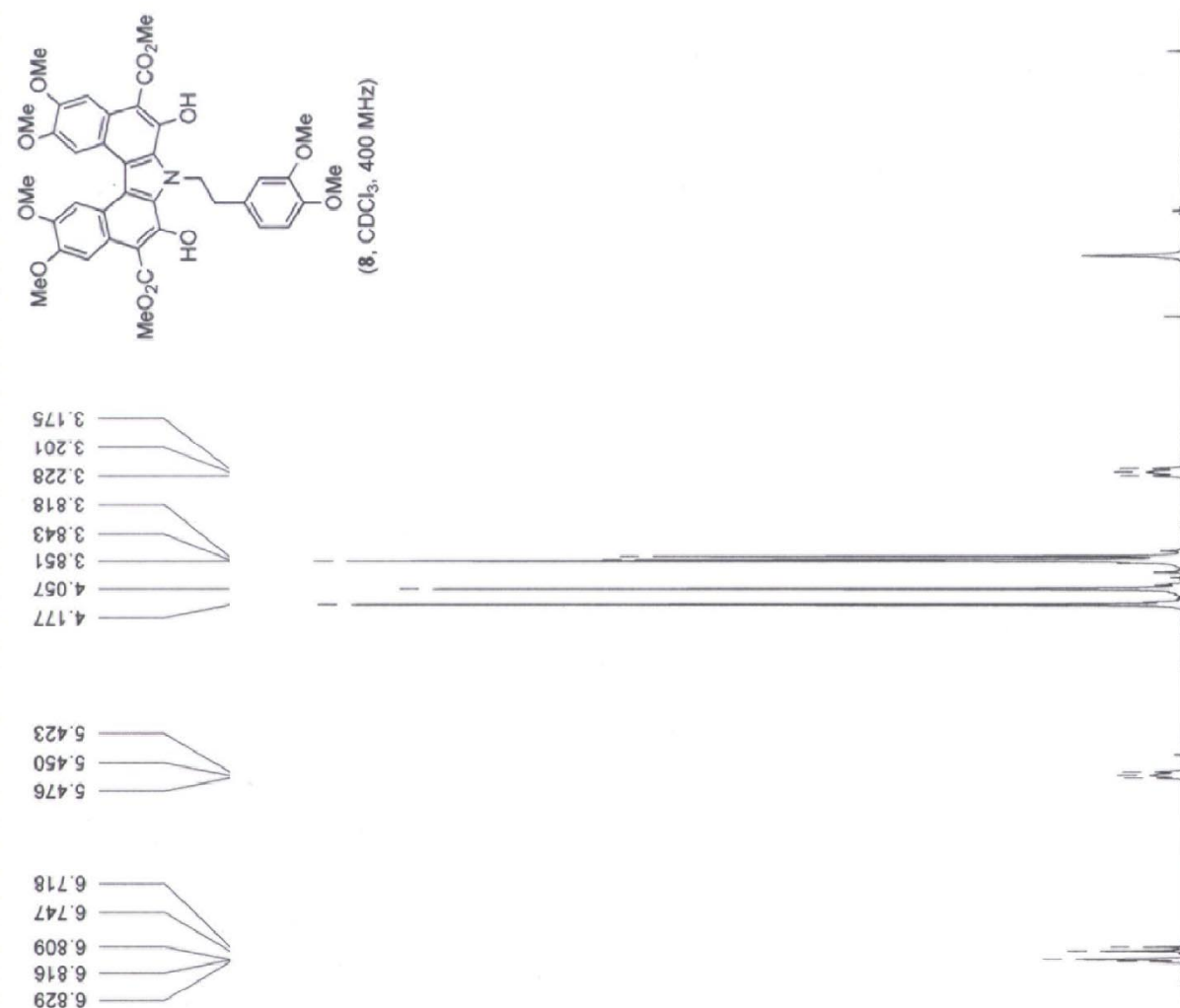

$680^{\circ}$

8928

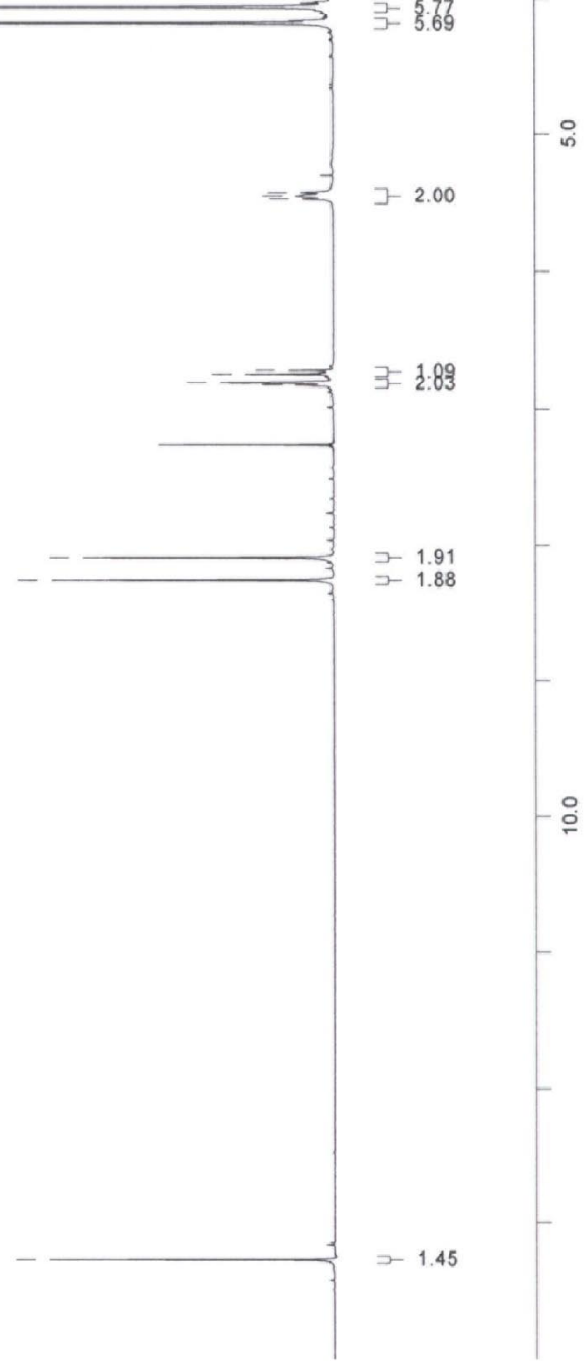



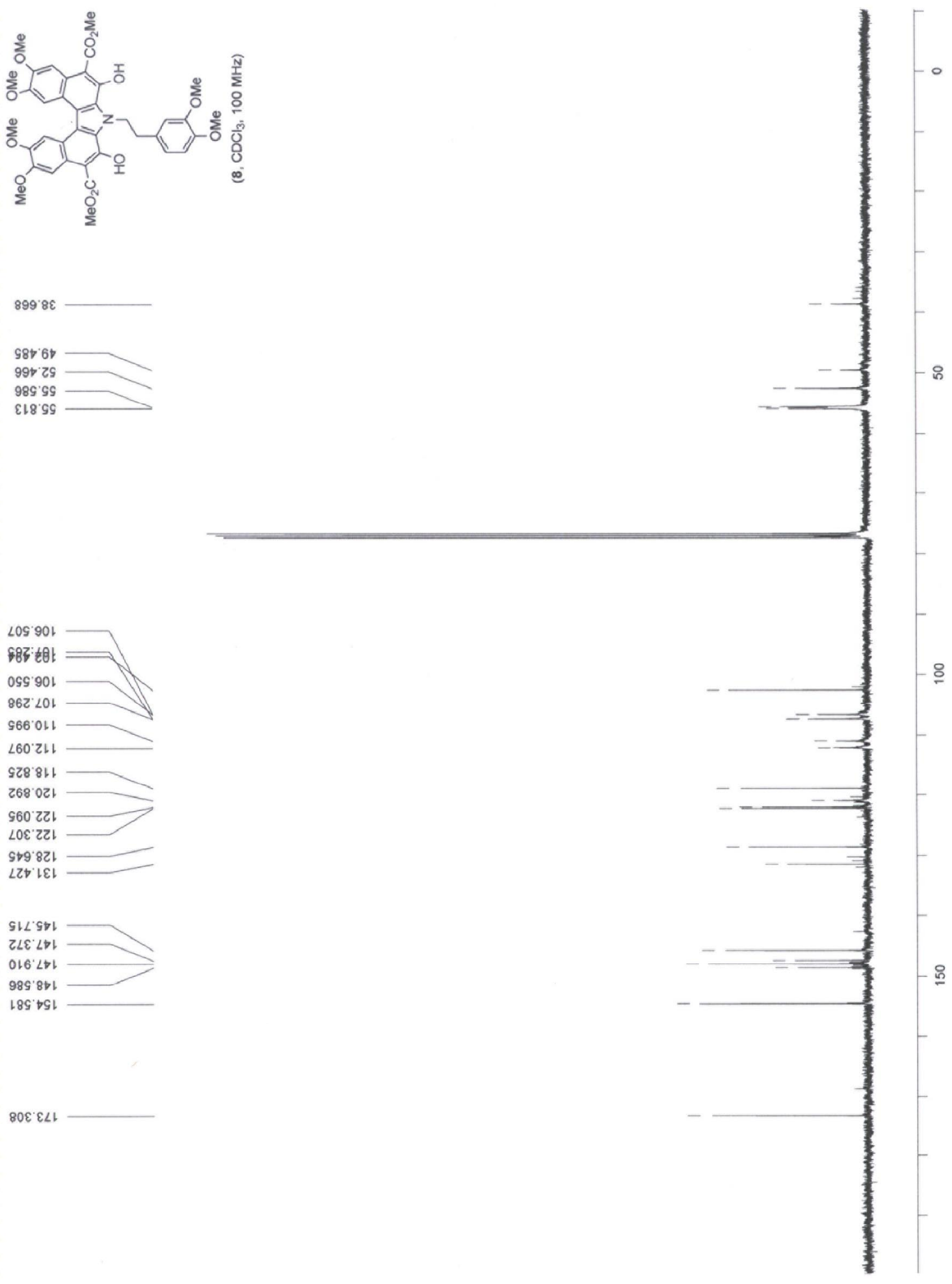

$80 \varepsilon^{\prime} \varepsilon<L$ 

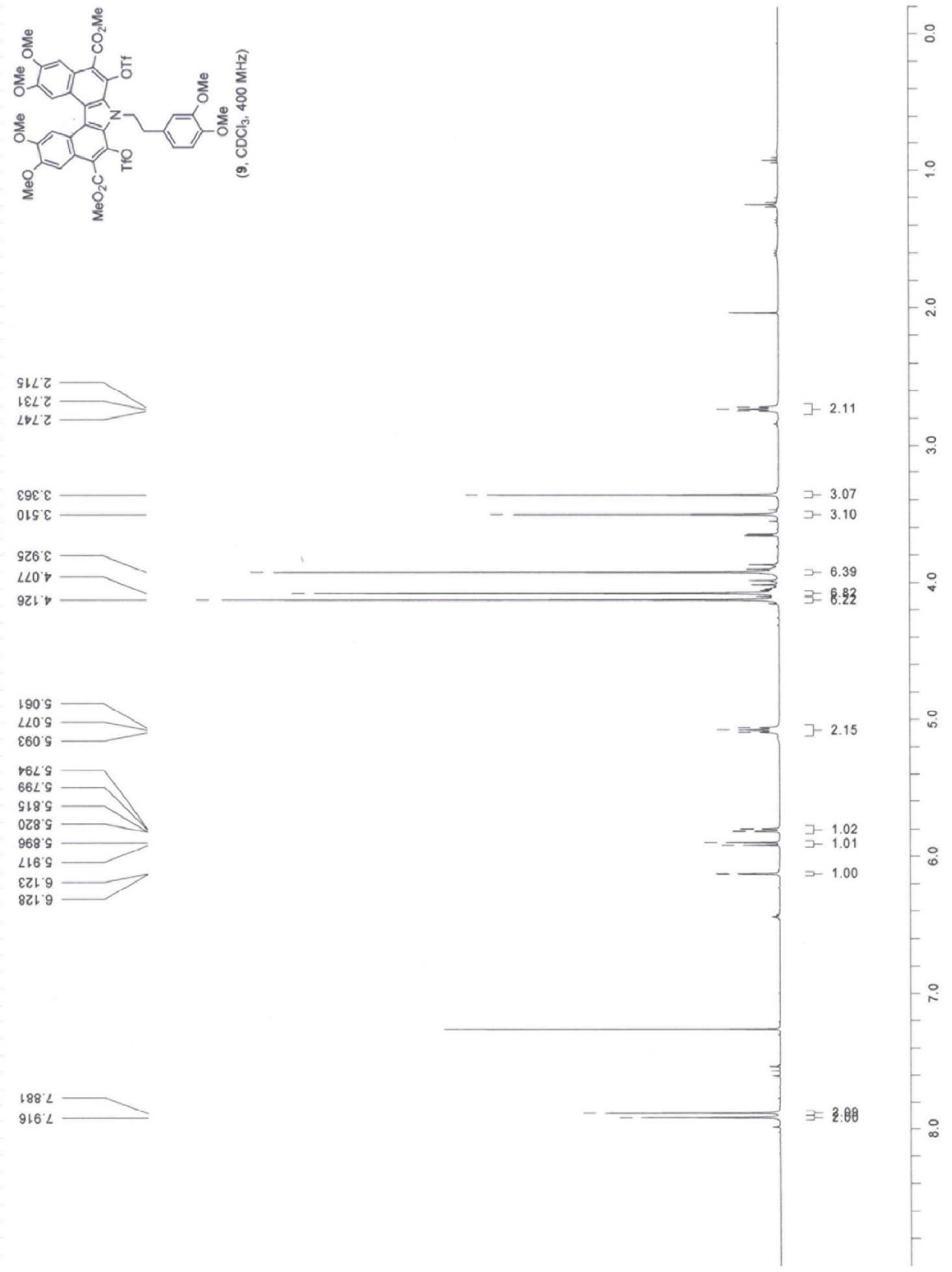

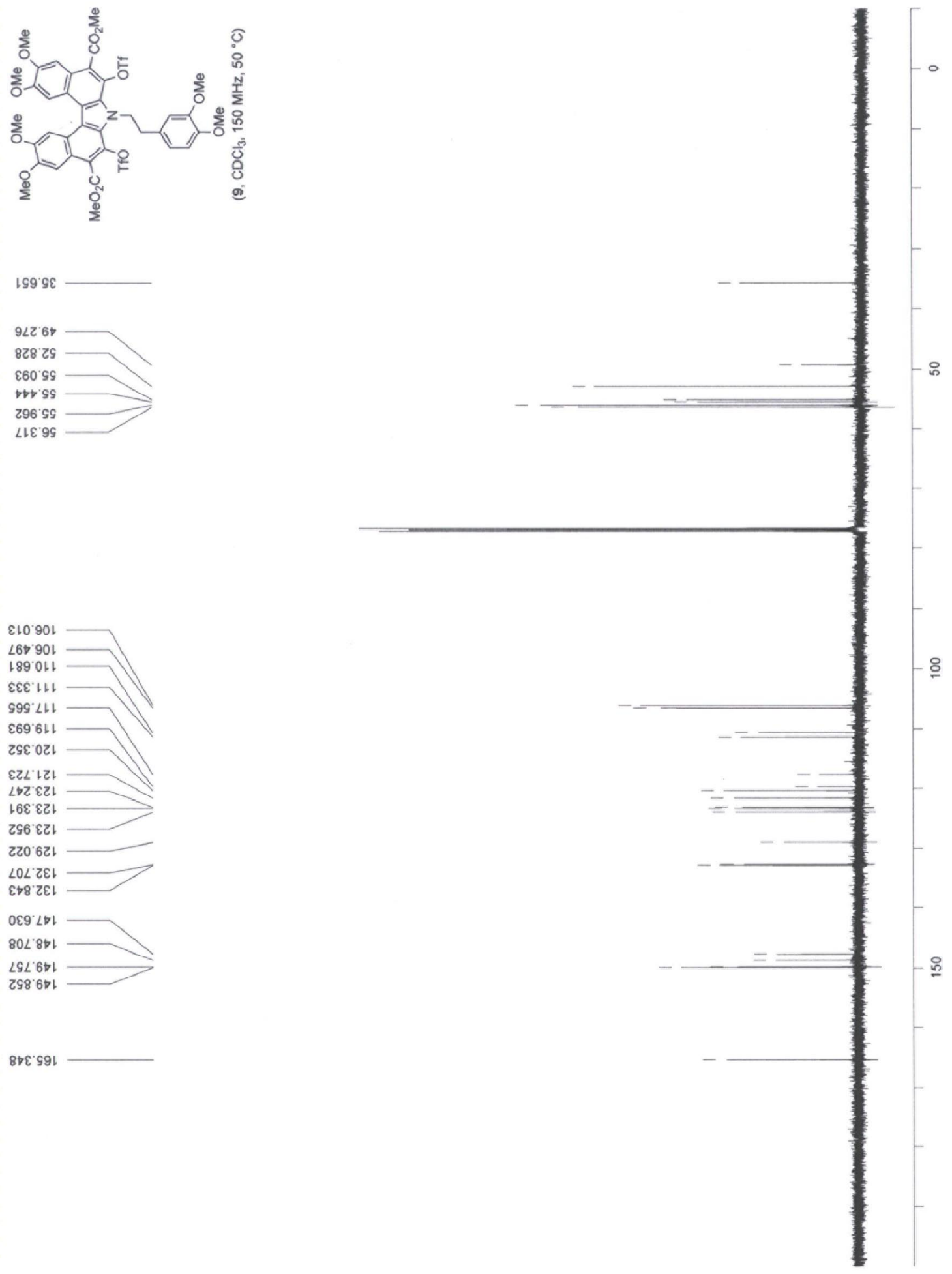

$8+\varepsilon$. 991 




แเว
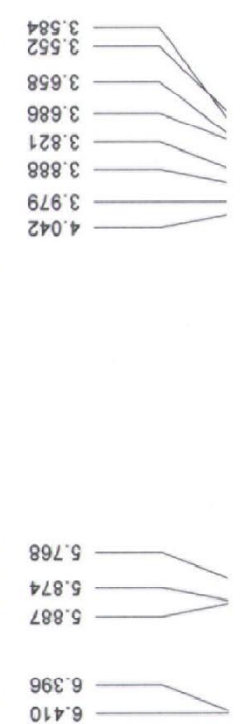

टL8.9

988

$9 \varepsilon 0^{\circ}$

$890^{\circ} \mathrm{L}$

$1 \angle 0 \% 2$

$12 \varepsilon^{\circ}$

$\varepsilon \mapsto \tau \cdot 8$

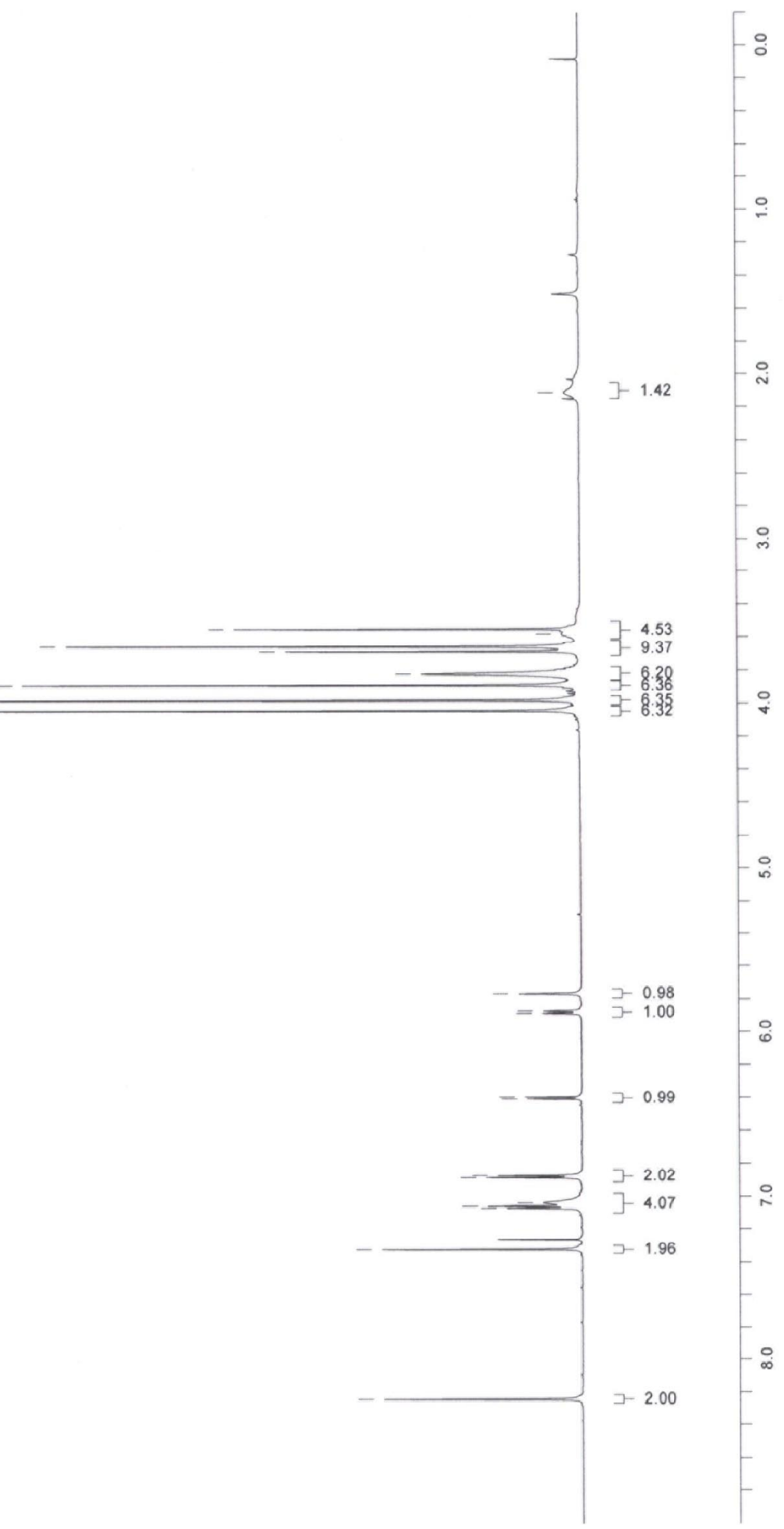



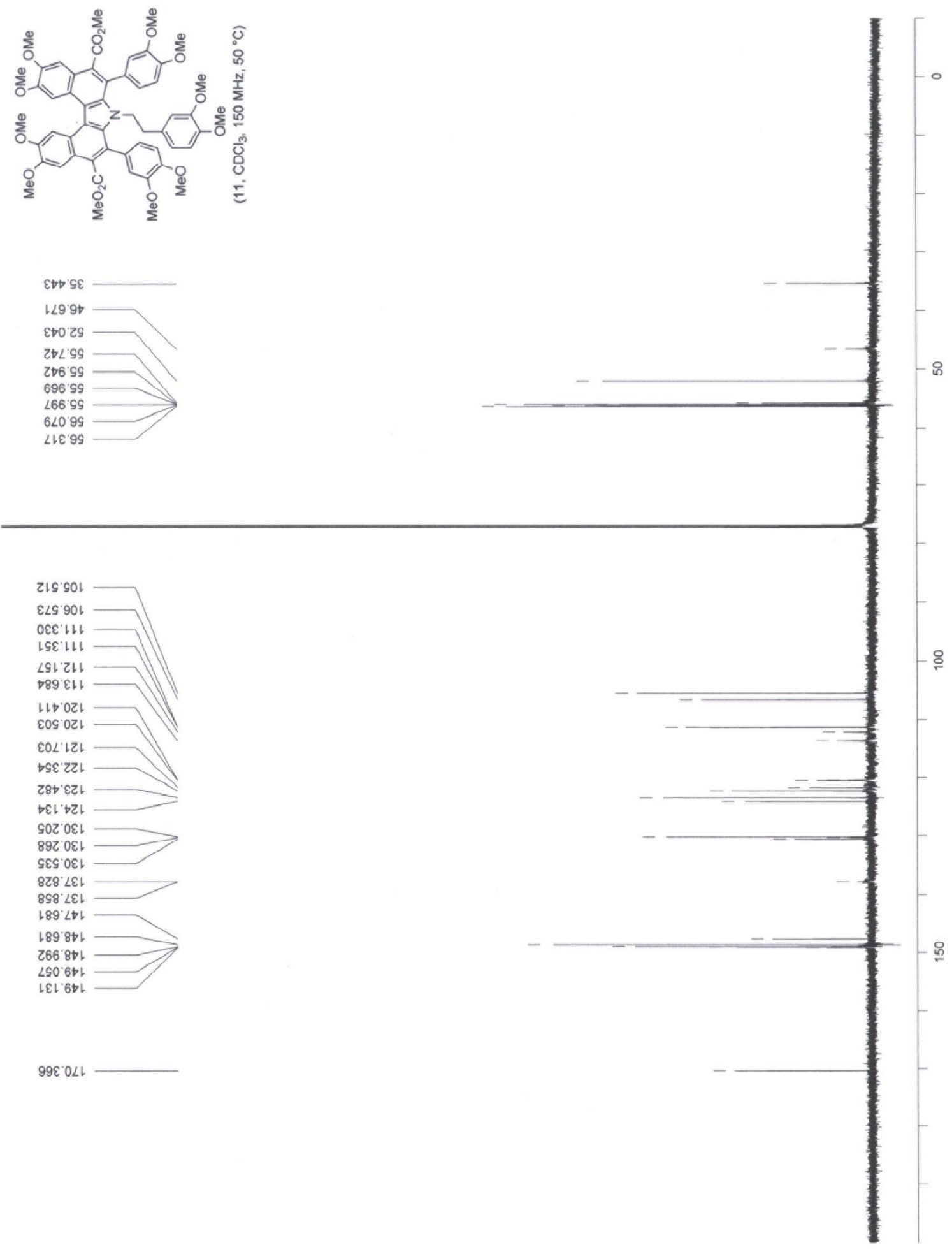

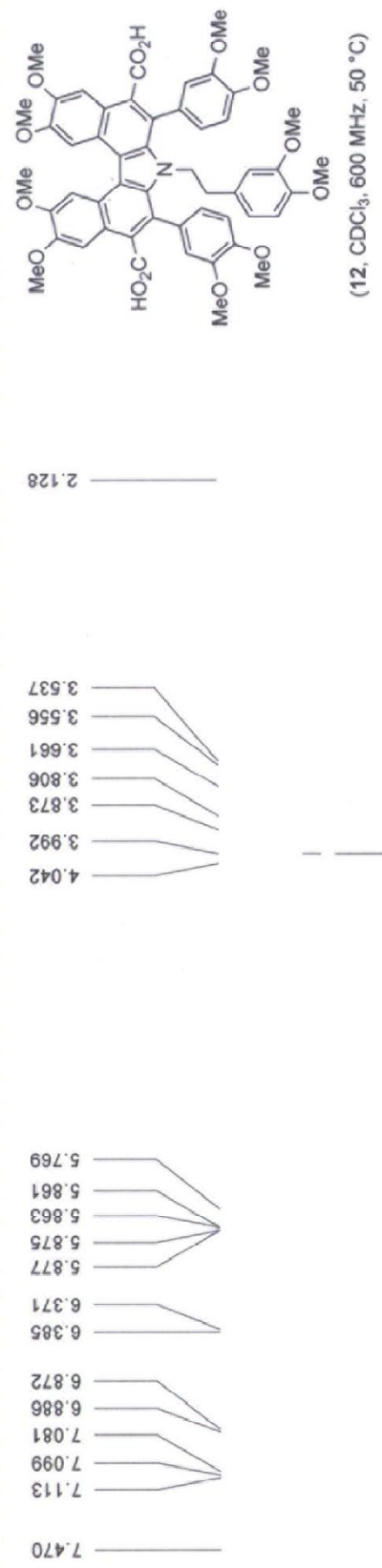

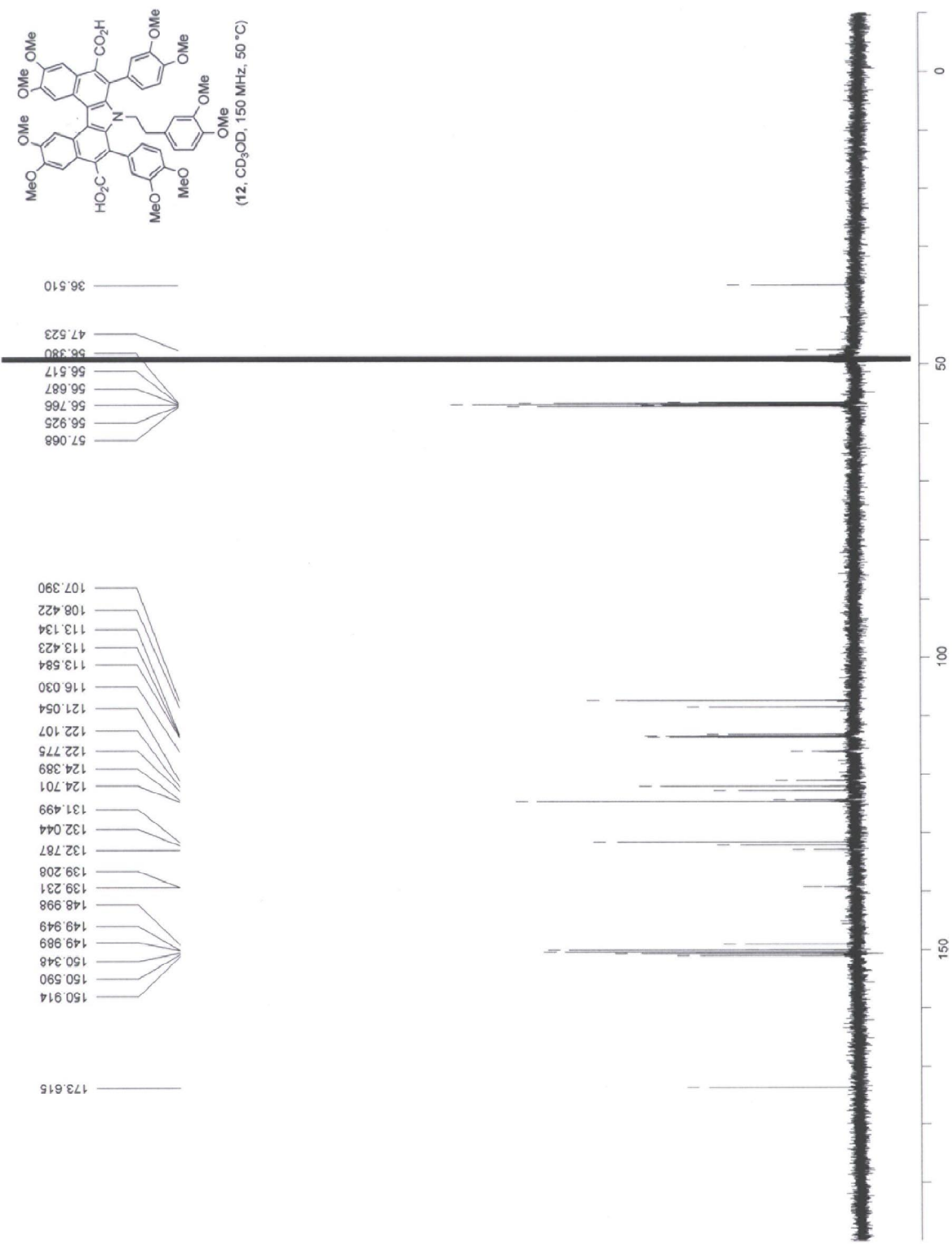

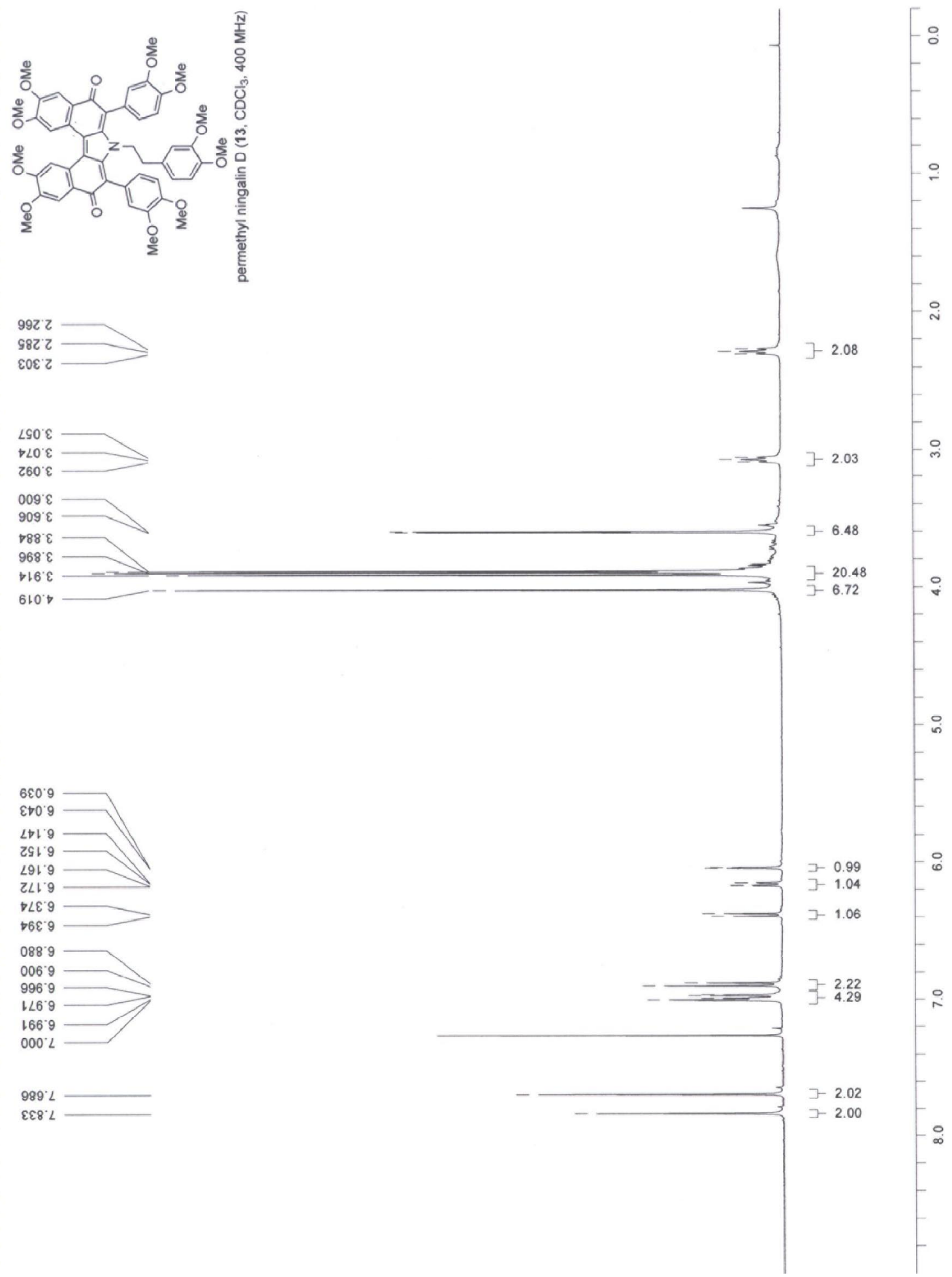

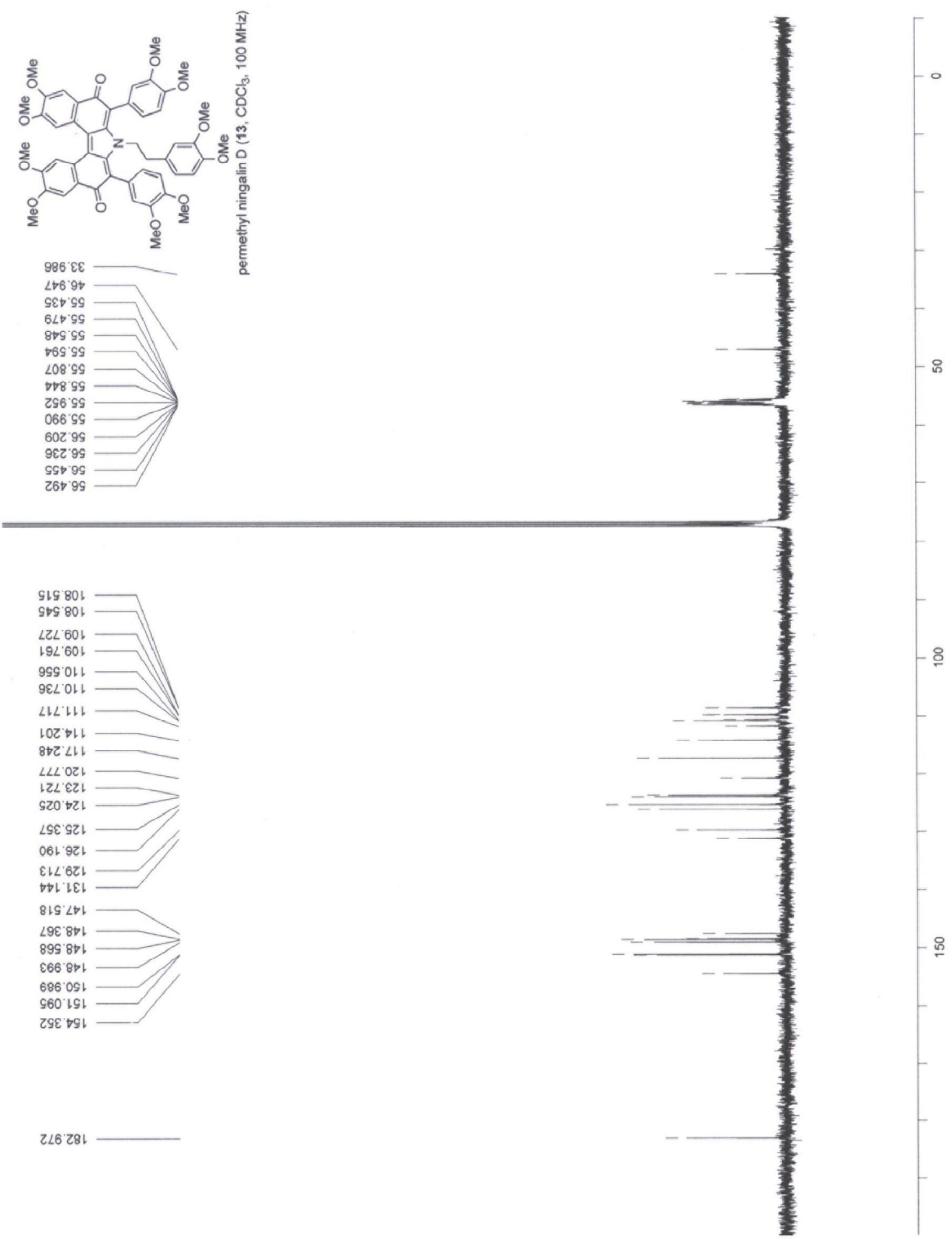

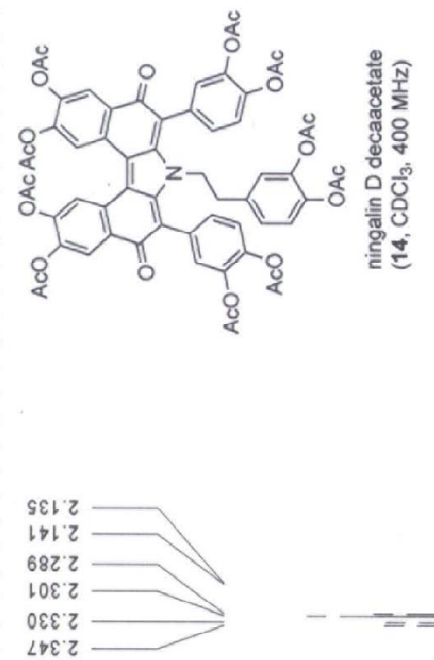

เย己
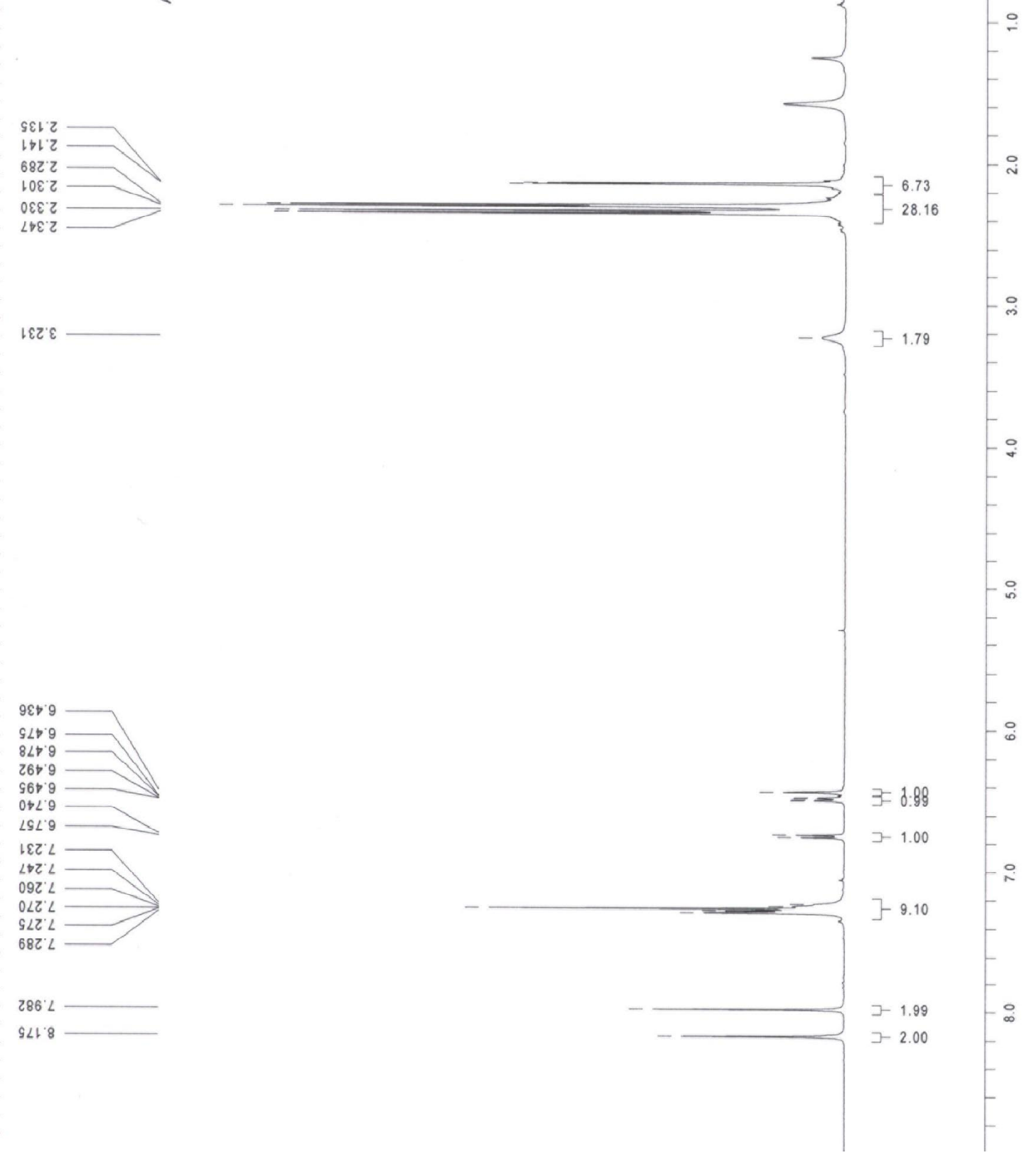


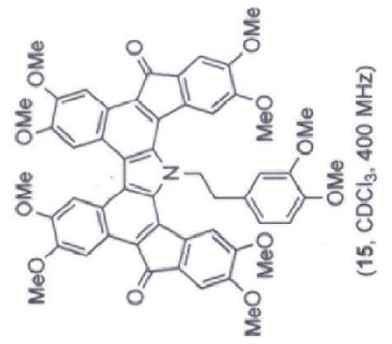

09\& 2

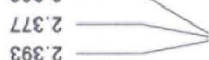

$\varepsilon 6 \varepsilon^{\prime} 乙$

$\varepsilon 6 เ \varepsilon$

$\varsigma ⿱ 一 \bullet \varepsilon-$

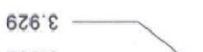

$286^{\circ} \mathcal{E}$

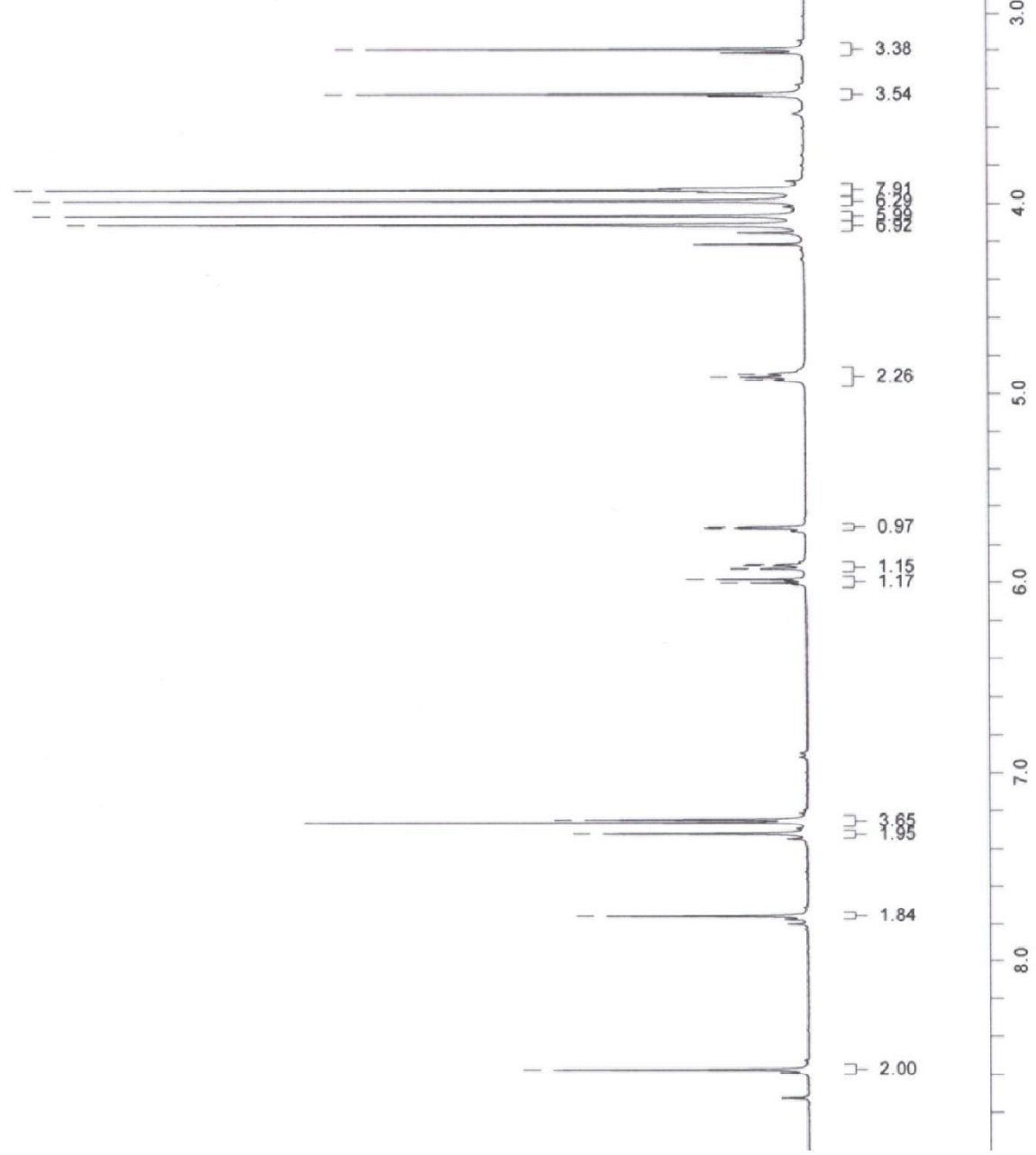

$\varepsilon 68$

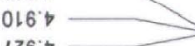

$\angle Z 6^{\circ} \circ \longrightarrow$

$80 L '$

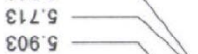

806.

ع 6 's

$826 \cdot 9$

286.5

$\varepsilon 00 \cdot 9$

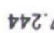

$61 \varepsilon^{2} 2$

$\forall G L ' L$

$1 \angle 9^{\circ} 8$ 


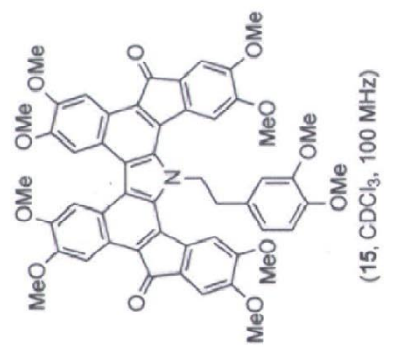

$8 \div 8 \varepsilon$

$9 \& 8$ ' $L S$
$9 \forall L \backslash S$

LEZ 99

EtL $S 9$

986 '

00p 99

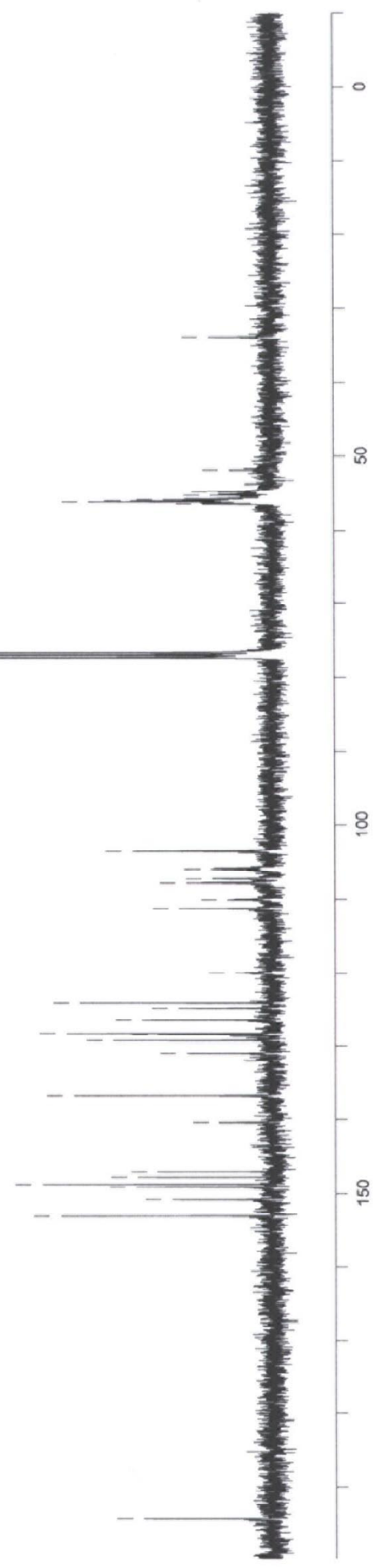




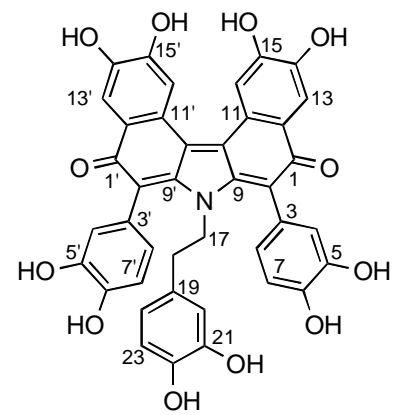

NMR Comparison of Synthetic and Natural 1a.

\begin{tabular}{|c|c|c|c|c|}
\hline C no. & ${ }^{13} \mathrm{C}$ : Reported & Observed & ${ }^{1} \mathrm{H}$ : Reported & Observed \\
\hline $1,1^{\prime}$ & 185.4 & 185.4 & & \\
\hline $2,2^{\prime}$ & 118.9 & 118.9 & & \\
\hline $3,3^{\prime}$ & 126.1 & 126.1 & & \\
\hline $4,4^{\prime}$ & 119.4 & 119.4 & $6.82(d)$ & 6.79-6.78 (overlap) \\
\hline $5,5^{\prime}$ & 146.1 & 146.2 & & \\
\hline $6,6^{\prime}$ & 146.5 & 146.6 & & \\
\hline $7,7^{\prime}$ & 116.2 & 116.3 & $6.83(d)$ & 6.79-6.78 (overlap) \\
\hline $8,8^{\prime}$ & 124.2 & 124.2 & $6.68(\mathrm{dd})$ & $6.64(\mathrm{dd})$ \\
\hline $9,9^{\prime}$ & 156.4 & 156.5 & & \\
\hline $10,10^{\prime}$ & 126.4 & 126.5 & & \\
\hline $11,11^{\prime}$ & 125.0 & 125.0 & & \\
\hline $12,12^{\prime}$ & 132.2 & 132.2 & & \\
\hline $13,13^{\prime}$ & 115.0 & 115.0 & $7.45(\mathrm{~s})$ & $7.41(\mathrm{~s})$ \\
\hline $14,14^{\prime}$ & 148.9 & 149.0 & & \\
\hline $15,15^{\prime}$ & 150.2 & 150.2 & & \\
\hline 16,16 & 114.2 & 114.2 & $7.85(\mathrm{~s})$ & $7.81(\mathrm{~s})$ \\
\hline 17 & 47.5 & 47.8 & $2.95(\mathrm{t})$ & $2.91(\mathrm{t})$ \\
\hline 18 & 34.5 & 35.0 & $2.13(\mathrm{t})$ & $2.09(\mathrm{t})$ \\
\hline 19 & 130.5 & 130.6 & & \\
\hline 20 & 117.1 & 117.2 & $6.12(d)$ & $6.08(d)$ \\
\hline 21 & 145.5 & 145.6 & & \\
\hline 22 & 144.6 & 144.7 & & \\
\hline 23 & 115.7 & 115.7 & $6.30(\mathrm{~d})$ & $6.26(d)$ \\
\hline 24 & 121.4 & 121.5 & $5.95(\mathrm{dd})$ & $5.91(\mathrm{dd})$ \\
\hline
\end{tabular}

